\title{
Absorption, autoionization, and predissociation in molecular hydrogen: High-resolution spectroscopy and multichannel quantum defect theory
}

\author{
Journal Article \\ Author(s): \\ Sommavilla, Marcel; Merkt, Frédéric; Mezei, J. Zs.; Jungen, Christian \\ Publication date: \\ 2016-02-28 \\ Permanent link: \\ https://doi.org/10.3929/ethz-a-010878978 \\ Rights / license: \\ In Copyright - Non-Commercial Use Permitted \\ Originally published in: \\ The Journal of Chemical Physics 144(8), https://doi.org/10.1063/1.4941920
}


This article may be downloaded for personal use only. Any other use requires prior permission of the author and AIP Publishing.

The following article appeared in J. Chem. Phys. 144(8), 084303 (2016) and may be found at http://dx.doi.org/10.1063/1.4941920. 
Absorption, autoionization, and predissociation in molecular hydrogen: Highresolution spectroscopy and multichannel quantum defect theory

M. Sommavilla, F. Merkt, J. Zs. Mezei, and Ch. Jungen'

Citation: J. Chem. Phys. 144, 084303 (2016); doi: 10.1063/1.4941920

View online: http://dx.doi.org/10.1063/1.4941920

View Table of Contents: http://aip.scitation.org/toc/jcp/144/8

Published by the American Institute of Physics 


\title{
Absorption, autoionization, and predissociation in molecular hydrogen: High-resolution spectroscopy and multichannel quantum defect theory
}

\author{
M. Sommavilla, ${ }^{1}$ F. Merkt, ${ }^{1}$ J. Zs. Mezei, ${ }^{2,3}$ and Ch. Jungen ${ }^{3, a)}$ \\ ${ }^{1}$ Laboratorium für Physikalische Chemie, ETH-Zürich, 8093 Zürich, Switzerland \\ ${ }^{2}$ Laboratoire Ondes et Milieux Complexes, UMR 6294 CNRS and Université du Havre, 25, rue Philippe Lebon, \\ BP 540, F-76058 Le Havre, France \\ ${ }^{3}$ Laboratoire Aimé Cotton du CNRS, Bâtiment 505, Université de Paris-Sud, F-91405 Orsay, France
}

(Received 16 December 2015; accepted 21 January 2016; published online 24 February 2016)

\begin{abstract}
Absorption and photoionization spectra of $\mathrm{H}_{2}$ have been recorded at a resolution of 0.09 and 0.04 $\mathrm{cm}^{-1}$, respectively, between $125600 \mathrm{~cm}^{-1}$ and $126000 \mathrm{~cm}^{-1}$. The observed Rydberg states belong to series $(n=10-14)$ converging on the first vibrationally excited level of the $\mathrm{X}^{2} \Sigma_{\mathrm{g}}^{+}$state of $\mathrm{H}_{2}^{+}$, and of lower members of series converging on higher vibrational levels. The observed resonances are characterized by the competition between autoionization, predissociation, and fluorescence. The unprecedented resolution of the present experimental data leads to a full characterization of the predissociation/autoionization profiles of many resonances that had not been resolved previously. Multichannel quantum defect theory is used to predict the line positions, widths, shapes, and intensities of the observed spectra and is found to yield quantitative agreement using previously determined quantum defect functions as the unique set of input parameters. (O) 2016 AIP Publishing LLC. [http://dx.doi.org/10.1063/1.4941920]
\end{abstract}

\section{INTRODUCTION}

Since the early days of molecular spectroscopy, the absorption spectrum of diatomic hydrogen has been studied and used to test the predictions of quantum mechanics. For instance, accurate experimental values for the dissociation and ionization energies of $\mathrm{H}_{2}$ and its isotopomers were derived in the early 1970s in Herzberg's group from its detailed study 1,2 and were compared with values obtained from ab initio quantum computations a few years earlier. ${ }^{3,4}$ In the same years the first highly resolved absorption spectra $\left(\leq 1 \mathrm{~cm}^{-1}\right)$ of $\mathrm{H}_{2}$, showing Rydberg series up to high principal quantum numbers $(n \approx 30)$, were obtained in the laboratory. ${ }^{2,5}$ Some of the lines in these spectra exhibited characteristic broadenings which were attributed to the combined effects of autoionization and predissociation. ${ }^{2,6}$ These decay mechanisms and their competition were experimentally characterized and quantified a few years later when Dehmer and Chupka published their pioneering study of photoabsorption, photoionization, and predissociation of $\mathrm{H}_{2}$ near the ionization threshold. ${ }^{7}$ The spectral resolution which they attained, however, was by about a factor 2 to 3 worse than that achieved in the photoabsorption spectra which Herzberg had recorded on photographic plates. Dehmer and Chupka nevertheless were in a position to resolve many resonances and observe them in different decay channels, and hence obtain information on the decay dynamics, which in turn they could relate to the observed resonance profiles.

In more recent years research on the absorption spectrum of diatomic hydrogen has followed two strands, one focussed

\footnotetext{
a)Also at Department of Physics and Astronomy, University College London, London WC1E 6BT, United Kingdom.
}

primarily on improving the spectral resolution of the experiments and the other aiming at a quantitative and systematic description of the excitation and decay dynamics. The work of Rothschild et al. ${ }^{8}$ and of Dickenson et al. ${ }^{9}$ belongs to the first strand. In the synchrotron experiment of Ref. 9, for instance, the absolute accuracy could be improved to $\approx 0.06 \mathrm{~cm}^{-1}$, but despite cooling the spectral resolution was still limited by a Doppler width of $0.7 \mathrm{~cm}^{-1}$. Along the other line of work, Glass-Maujean and her collaborators ${ }^{10-12}$ succeeded, over the years, to produce an essentially complete and accurate account of the decay dynamics of superexcited $\mathrm{H}_{2}$, including fluorescence in addition to dissociation and ionization, and extending from the ionization threshold nearly two $\mathrm{eV}$ into the continuum. However, the resolution of their synchrotron studies carried out at Bessy II in Berlin is limited by the Doppler effect at room temperature and their absolute accuracy corresponds to $\approx 0.8 \mathrm{~cm}^{-1}$, and therefore only the broader resonances could be mapped out in detail.

We also mention the double-resonance laser studies combined with electron spectroscopy carried out in the 1980s and 1990s on $\mathrm{H}_{2}$, in which ungerade levels subject to autoionization and predissociation were observed in excitation from the $E, F^{1} \Sigma_{g}^{+}$state (see references given in Ref. 13). While in these experiments the energy of the photoelectrons could be measured and hence branching ratios could be determined, the effective resolution width remained limited to $\approx 0.7 \mathrm{~cm}^{-1}$, and sharp intense transitions were saturated. Also, at that time no efficient theoretical multichannel quantum defect theory (MQDT) treatment of predissociation was available. ${ }^{13}$

In this work, we report absorption spectra of $\mathrm{H}_{2}$ recorded using a molecular jet which are essentially Doppler-free and correspond to an effective spectral resolution of $\approx 0.09 \mathrm{~cm}^{-1}$ (absorption) and $\approx 0.04 \mathrm{~cm}^{-1}$ (photoionization). We have 
recorded absorption and photoionization spectra in the region between the $v^{+}=1$ and $v^{+}=2$ vibrational ionization limits of the $\mathrm{H}_{2}^{+}$ion, in which numerous resonances have been mapped out in unprecedented detail. Our results are interpreted with the help of MQDT implemented here on the basis of ab initio input data without any fit to experiment, in a MQDT approach which accounts for dissociation and ionization processes as well as their competition.

It has been known for a long time ${ }^{2}$ that to the extent that spin effects and $\ell$ mixing may be neglected, the excited $p$ states of $\mathrm{H}_{2}$ behave very differently depending on whether they are derived from ${ }^{1} \Sigma_{u}^{+1}, \Pi_{u}^{+}$or from ${ }^{1} \Pi_{u}^{-}$electronic symmetry. In $\mathrm{H}_{2}$ ground state absorption, the ${ }^{1} \Pi_{u}^{-}$levels that have total parity $-(-1)^{N}$ (so-called $d$ parity levels, $\left.N^{d 14}\right)$ are excited via $Q\left(N=N^{\prime \prime}\right)$ electric dipole transitions. Here $N^{\prime \prime}$ and $N$ are, respectively, the initial and final state total angular momentum exclusive of spin. The ${ }^{1} \Sigma_{u}^{+},{ }^{1} \Pi_{u}^{+}$ levels have total parity $+(-1)^{N}$ (so-called $c$ parity levels, $\left.N^{c 14}\right)$ and are excited via $P\left(N^{\prime \prime}=N+1\right)$ and $R\left(N^{\prime \prime}=N-1\right)$ transitions. The $N^{d}$ components of the $p$ manifolds exhibit no rotational-electronic $\ell$ uncoupling interaction and are subject to only weak vibronic interactions and hence slow autoionization and predissociation. The $N^{c}$ symmetry levels on the other hand produce Rydberg series that occur in pairs, which owing to rotational-electronic interaction evolve from Hund's case $b n p \Sigma$ and $n p \Pi$ states into Hund's case $d$ series $n p N^{+}$converging to the rotational ion thresholds $N^{+}=N \pm 1$ (i.e., $\vec{N}=\vec{N}^{+}+\vec{\ell}$ ). These states autoionize and predissociate rapidly and are heavily perturbed by rovibronic interactions. Because of the high resolution which we achieve here, the different behavior of the two classes of symmetry levels in $\mathrm{H}_{2}$ absorption and photoionization is revealed particularly clearly in our absorption and photoionization spectra.

The $\mathrm{H}_{2}$ resonances produced by predissociation and autoionization, which are the subject of the present paper, belong to a class commonly referred to as "Feshbach" resonances, as opposed to "shape resonances"15 which correspond to quasi-bound levels decaying through a potential barrier. The present "Feshbach" resonances are due to the molecular Hamiltonian terms coupling the electronic and nuclear degrees of freedom, which are neglected in the Born-Oppenheimer approximation. Broad Feshbach-type resonances occur in $\mathrm{H}_{2}$ also in the gerade manifold of electronic states (which are not excited by absorption of a single photon), but these are due to electronic interactions between singly and doubly excited electron configurations. $^{16}$

\section{EXPERIMENT}

The experiments were carried out using a vacuumultraviolet (VUV) laser system and a spectrometer designed for the simultaneous recording of VUV absorption and photoionization spectra of atoms or molecules in skimmed supersonic beams. The experimental procedure and the apparatus have been described in Ref. 17, and we only give a brief overview here, with emphasis on the modifications that have been made to improve the sensitivity of the absorption measurements.

Pulsed, tunable radiation with wave number $\tilde{v}_{V U V}$ $=2 \tilde{v}_{1}+\tilde{v}_{2}$ was produced at a repetition rate of $25 \mathrm{~Hz}$ by resonant four-wave mixing in a pulsed beam of krypton using the $(4 \mathrm{p})^{5} 5 \mathrm{p}[1 / 2]_{0} \leftarrow(4 \mathrm{p})^{6}$ two-photon resonance of $\mathrm{Kr}$, as described in Ref. 18. The laser beams with wave numbers $\tilde{v}_{1}$ and $\tilde{v}_{2}$ were obtained by pulsed amplification of the singlemode outputs of two continuous-wave ring dye lasers in dye cells pumped by the second harmonic of a pulsed Nd:YAG laser and subsequent frequency doubling $\left(\tilde{v}_{2}\right)$ and tripling $\left(\tilde{v}_{1}\right)$ in $\beta$-barium-borate crystals. To calibrate the VUV wave number, the wave number of the continuous-wave output of the first ring dye laser was stabilized to $15682.1511(17) \mathrm{cm}^{-1}$, yielding a value of $94092.907(10) \mathrm{cm}^{-1}$ for $2 \tilde{v}_{1}$, and the wave number of the second laser was calibrated using a simultaneously recorded spectrum of molecular iodine, as described in Ref. 19. The overall uncertainty of the VUV wave number was $0.02 \mathrm{~cm}^{-1}$, which includes a small contribution arising from the frequency chirp induced by the pulseamplification and the nonlinear frequency up-conversion processes. The VUV laser beam was separated from the fundamental beams and beams generated in other nonlinear optical processes using a grating monochromator. The VUV beams diffracted in the positive and negative first order were used as probe (intensity $I$ ) and reference (intensity $I_{0}$ ) beams, respectively. Between the monochromator grating and the detector used to monitor the VUV-laser pulse energies, the probe beam traversed two chambers, a photoionization chamber and an absorption chamber (see lower left part of Fig. 1).

In the photoionization chamber, the VUV probe beam crossed a supersonic beam of pure $\mathrm{H}_{2}$ at right angles on the axis of a time-of-flight mass spectrometer. The low background pressure $\left(\leq 10^{-6} \mathrm{mbar}\right)$ needed for optimal performance of the time-of-flight mass spectrometer was achieved by placing a skimmer with a $500 \mu \mathrm{m}$ orifice diameter about $10 \mathrm{~cm}$ downstream from the orifice of the pulsed valve used to generate the beam and about $6 \mathrm{~cm}$ upstream from the intersection point with the VUV probe beam. To enhance the absorption signal, the VUV probe beam traversed another ten skimmed supersonic beams in the absorption chamber before reaching the VUV detector (see upper right part of Fig. 1). The reduced distance of $3.5 \mathrm{~cm}$ between the valves and the skimmers and the larger skimmer-orifice diameter $(1 \mathrm{~mm})$ enabled a more than 20-fold increase of the column density and thus of the sensitivity of the absorption measurements. Differential pumping between the chambers and between the regions on either sides of the skimmers maintained a background pressure of about $\left(2-5 \cdot 10^{-7} \mathrm{mbar}\right)$ in the monochromator and in the tube through which the reference VUV beam propagated before reaching the second VUV detector.

At each frequency step $i$ of the scans, both the photoionization and the transmission signals were determined. The transmission signal $\tau_{i}$ was obtained by building the ratio $\tau_{i}=I_{i} / I_{i, 0}$, which enabled the suppression of the noise arising from pulse-to-pulse fluctuations of the VUV-laser intensity. The photoionization signal was determined by extracting the 


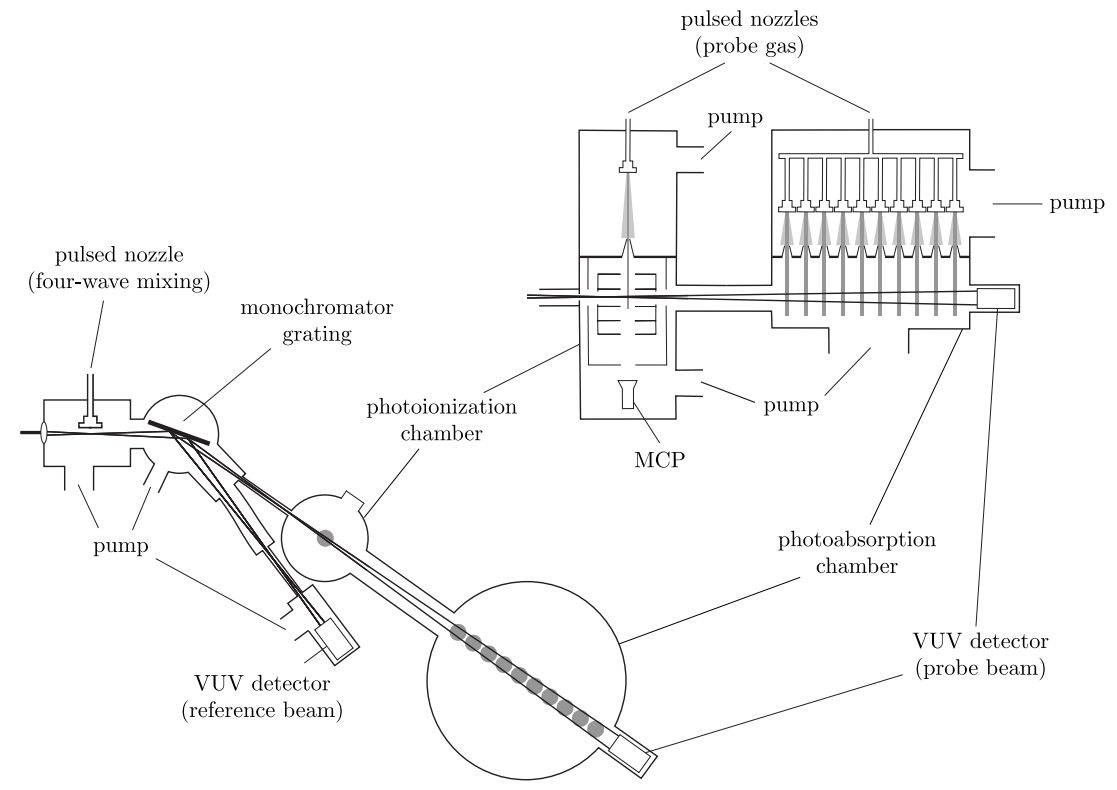

FIG. 1. Schematic diagram of the spectrometer used to simultaneously record VUV photoionization and absorption spectra of molecular hydrogen.

ions with pulsed potentials applied to a set of cylindrical metallic plates surrounding the photoexcitation region and monitoring the $\mathrm{H}_{2}^{+}$ion current with a microchannel-plate detector located at the end of a 15-cm-long flight tube.

The alignment and relative timing of the VUV-laser and gas beams in the absorption chamber were optimized by successively monitoring and maximizing the absorption resulting from each of the ten pulsed nozzles using a strong transition of argon, the $(3 p)^{5} 9 \mathrm{~d}[3 / 2]_{1} \leftarrow(3 p)^{6}$ transition, which is located in the immediate vicinity of the spectral region of interest. The result of the optimization procedure is illustrated in Fig. 2 which compares, in the upper panel, the absorption spectrum obtained with a single pulsed valve (full line) with that obtained with all 10 valves in operation (dashed line). The figure illustrates that about $75 \%$ of the VUV intensity was absorbed by a single argon beam at the central position (125718.08(3) $\mathrm{cm}^{-1}$ ) of the resonance of the ${ }^{40} \mathrm{Ar}$ isotope. After optimization, each beam absorbed at least $70 \%$ of the VUV intensity, some even more than $80 \%$. When the ten valves were in operation, the absorption line of ${ }^{40} \mathrm{Ar}$ was strongly broadened by saturation, and the transition of the ${ }^{36} \mathrm{Ar}$ isotope (natural abundance of $0.337 \%$ ) became observable at $125717.98(3) \mathrm{cm}^{-1}$. Resonance-enhanced $\left(1+1^{\prime}\right)$ twophoton-ionization spectra of the three natural isotopes of $\mathrm{Ar}$ were recorded simultaneously and are depicted in the lower panel of Fig. 2 for comparison. The line widths (full width at half maximum (FWHM) of $0.02 \mathrm{~cm}^{-1}$ ) of the absorption lines are slightly broader than those of the lines of the photoionization spectrum (full width at half maximum of $0.012 \mathrm{~cm}^{-1}$ ) because of the larger Doppler width resulting from the reduced valve-to-skimmer distance and the larger orifice diameter of the skimmers used in the absorption chamber.

During operation of the nozzles, the background pressure in the absorption chamber rose above $10^{-6} \mathrm{mbar}$, which caused a measurable absorption by the background room-temperature Ar gas in the chamber. In the spectrum displayed in the bottom panel of Fig. 3, which was obtained with only one nozzle,

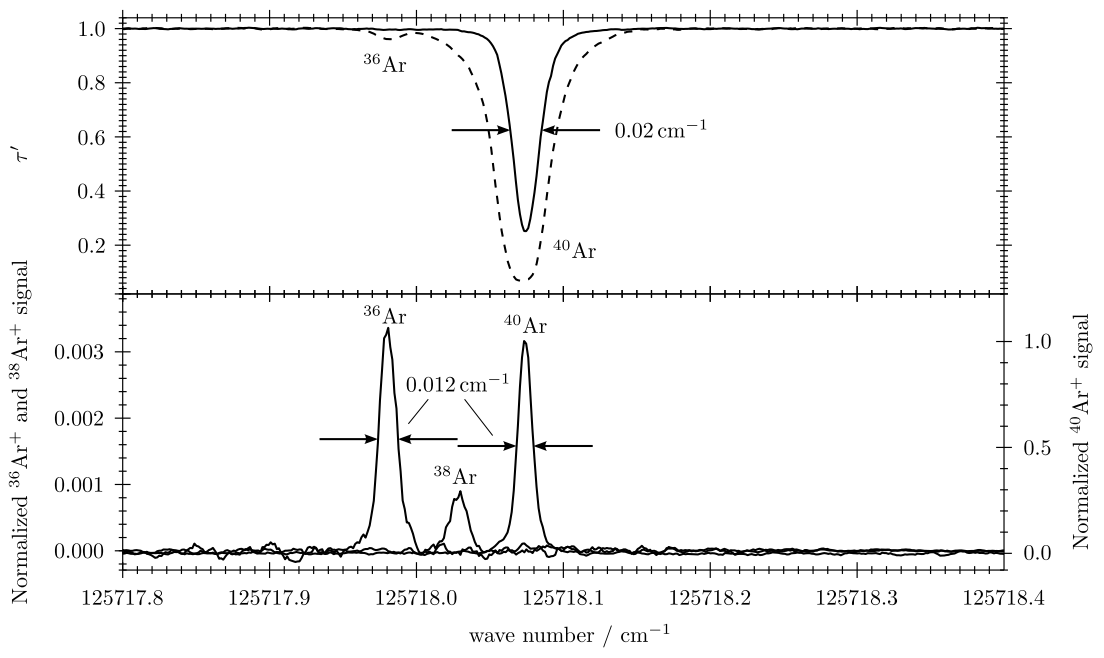

FIG. 2. Absorption (upper panel) and $\left(1+1^{\prime}\right)$ resonance-enhanced two-photon-ionization (lower panel) spectra of $\mathrm{Ar}$ in the vicinity of the $(3 p)^{5} 9 \mathrm{~d}[3 / 2]_{1} \leftarrow(3 p)^{6} \quad$ transition. In the upper panel, the full and dashed lines correspond to spectra recorded by operating a single nozzle and ten nozzles in the absorption chamber, respectively. In the lower panel, the traces labeled ${ }^{36} \mathrm{Ar},{ }^{38} \mathrm{Ar}$, and ${ }^{40} \mathrm{Ar}$ were obtained by monitoring the ionization signal at the corresponding masses in the time-of-flight spectrum as a function of the VUV wave number. Ionization from the $(3 p)^{5} 9 \mathrm{~d}[3 / 2]_{1}$ level was achieved using the second harmonic $(532 \mathrm{~nm})$ of the Nd:YAG laser used to pump the dye amplification cells. 


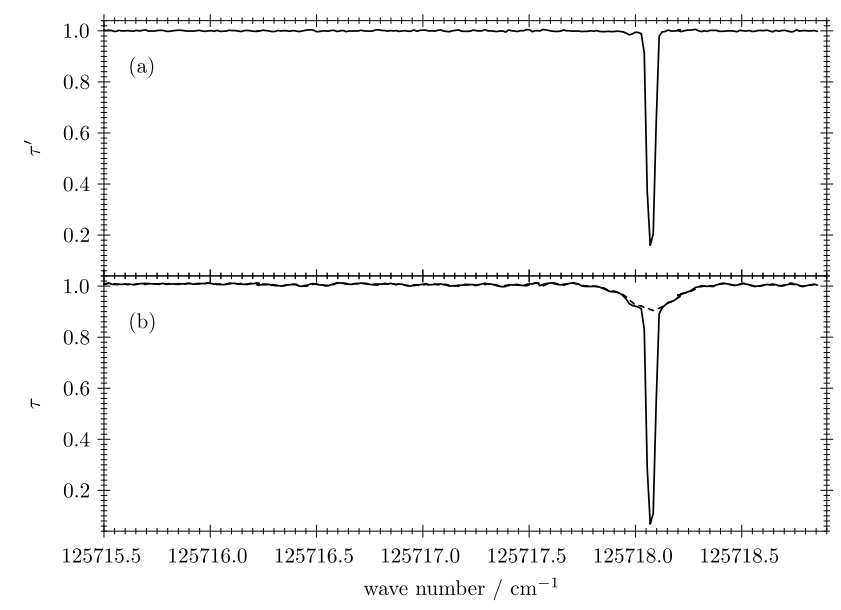

FIG. 3. Procedure used to remove the absorption by the thermal background gas in the chamber. (a) VUV absorption spectrum of Ar after background gas correction and corresponding to $\tau_{i}^{\prime}$ in Eq. (1). (b) Transmission signal measured with optimal temporal overlap of the VUV laser and probe-gas beams (full line) and with no temporal overlap between the probe-gas and VUV laser beams (dashed line).

this absorption by the background gas is noticeable as a broad pedestal with a width corresponding to the Doppler width of the argon gas at room-temperature. To eliminate this background absorption, the VUV intensity of the probe and reference gas beams were measured twice at each frequency step $i$, once with optimal temporal overlap of the VUV-laser and probe-gas beams $\left(I_{i}, I_{i, 0}, \tau_{i}=I_{i} / I_{i, 0}\right.$; see full line in the bottom panel of Fig. 3) and once with no temporal overlap between the VUV-laser and probe-gas beams $\left(I_{i}^{(\mathrm{b})}\right.$, $I_{i, 0}^{(\mathrm{b})}, \tau_{i}^{(\mathrm{b})}=I_{i}^{(\mathrm{b})} / I_{i, 0}^{(\mathrm{b})}$; “(b)" for background; see dashed line in the bottom panel of Fig. 3). The transmission signal $\tau_{i}^{\prime}$ corrected for absorption by the background gas is displayed in the top panel of Fig. 3 and was obtained using

$$
\tau_{i}^{\prime}=1+\left(\tau_{i}-\tau_{i}^{(\mathrm{b})}\right) /\left\langle\tau^{(\mathrm{b})}\right\rangle,
$$

where $\left\langle\tau^{(b)}\right\rangle$ represents the average value of the background absorption signal. The division by $\left\langle\tau^{(\mathrm{b})}\right\rangle$ in Eq. (1) guarantees that $\tau_{i}^{\prime}$ lies between 0 and 1 . After the background correction, the weak absorption line of ${ }^{36} \mathrm{Ar}$ is visible on the low-wavenumber side of the ${ }^{40} \mathrm{Ar}$ absorption line.

The absorption spectra of $\mathrm{H}_{2}$ presented in Sec. IV were corrected for the background-gas absorption using Eq. (1). To record these spectra, a pure $\mathrm{H}_{2}$ sample was used and the relative timing of the VUV laser pulse and the $\mathrm{H}_{2}$ gas pulse was modified to account for the higher velocity of the $\mathrm{H}_{2}$ supersonic beam $\left(v_{\mathrm{H}_{2}}=v_{\mathrm{Ar}} \sqrt{m_{\mathrm{Ar}} / m_{\mathrm{H}_{2}}}\right)$. The higher velocity of the $\mathrm{H}_{2}$ beam also resulted in an increased Doppler width in both the photoionization and absorption spectra, as will be discussed in more detail in Section IV. The column density of $\mathrm{H}_{2}$ molecules was estimated to be $1.1(4) \cdot 10^{13}$ molecule $/ \mathrm{cm}^{2}$ in a separate set of measurements of the photoionization cross section of Xe above the ${ }^{2} P_{1 / 2}$ threshold at $109688.7 \mathrm{~cm}^{-1}$ using the same apparatus and procedure, and correcting for the different expansion of $\mathrm{Xe}$ and $\mathrm{H}_{2}$ gas. Because of the removal from the absorption spectrum of the contribution from $\mathrm{H}_{2}$ molecules in the background gas, the absorption signal
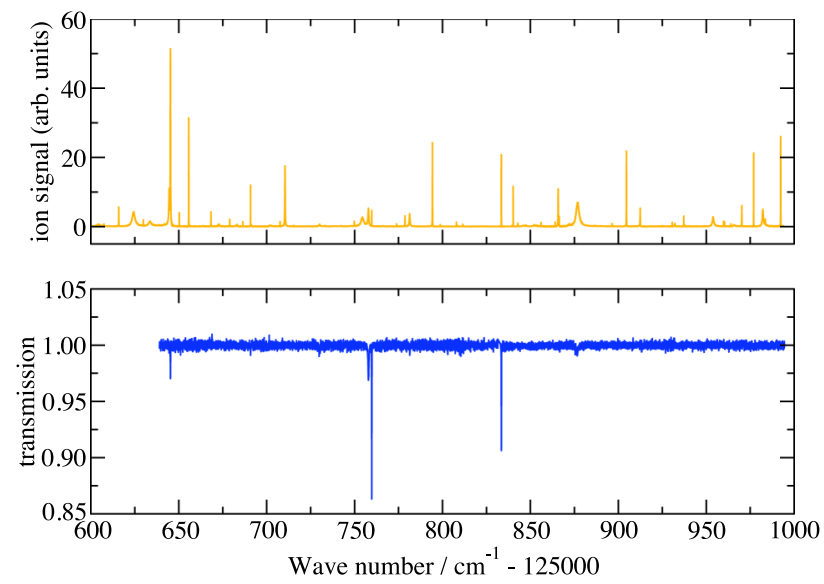

FIG. 4. Photoionization (upper panel) and transmission (lower panel) spectra of $\mathrm{H}_{2}$ for photon energies in the range $125600-126000 \mathrm{~cm}^{-1}$.

corresponds to a rotationally cold sample with population only in the $N^{\prime \prime}=0$ and 1 levels, with relative contributions of $25 \%$ and $75 \%$, respectively. Removal of the backgroundgas contribution from the photoionization spectrum could not be carried out, so that the photoionization spectrum consists of the sum of a cold component as described above for the absorption measurement and a component from the background gas thermalized to room-temperature in a ratio of about $0.4 / 0.6$, which gives relative populations in the $N^{\prime \prime}=0$, 1,2 , and 3 ground-state rotational levels of $18 \%, 72 \%, 6.7 \%$, and $2.6 \%$, respectively. Figure 4 presents an overview of the photoionization and transmission spectra of $\mathrm{H}_{2}$ obtained with the procedure just described.

\section{THEORETICAL CONSIDERATIONS}

\section{A. Multichannel quantum defect theory}

MQDT derives from standard scattering theory, extended such that in addition to continuum ("open") channels, discrete ("closed") channels also appear explicitly in the theory. ${ }^{20,21}$ This is not the case in the usual form of scattering theory where quasi-bound structures do not appear as independent actors in the multichannel formalism, but only indirectly via a resonant behavior of phase shifts and cross sections at certain energies. In the present work, we consider initially ion-electron scattering involving the $\mathrm{H}_{2}^{+} X^{+}$rovibrational target states $i=\left|v^{+} N^{+}\right\rangle$and the $\ell=1$ Rydberg electron, but we also address below (Sec. III E) the problem of reactive scattering where the charged $\mathrm{H}_{2}^{+}+\mathrm{e}^{-}$collision partners are converted into $\mathrm{H}(1 s)+\mathrm{H}(n=2)$ fragments.

To keep some degree of generality we denote the internal target (core) states $i$ by $\chi_{i}(q)$ with energies $E_{i}^{+}$, where $q$ refers to all coordinates of the system other than the radial coordinate $r$, thus including also the angular motion of the collision partners (electron partial wave $\ell$ ). For electron positions outside the molecular ion core a multichannel wavefunction then takes the general form of a double expansion over channels $i$ and $i^{\prime},{ }^{20,22}$ 


$$
\begin{aligned}
\Psi\left(E, q, r_{c}<r\right) & =\sum_{i^{\prime}} \Psi_{i^{\prime}}\left(E, q, r_{c}<r\right) B_{i^{\prime}}(E) \\
& =\sum_{i^{\prime}}\left\{\sum_{i} \chi_{i}(q) \frac{1}{r}\left[f_{i}\left(\epsilon_{i}, r\right) C_{i, i^{\prime}}(E)-g_{i}\left(\epsilon_{i}, r\right) \mathcal{S}_{i, i^{\prime}}(E)\right]\right\} B_{i^{\prime}}(E) .
\end{aligned}
$$

Here $f(\epsilon, r)$ and $g(\epsilon, r)$ represent a linearly independent energy-normalized base pair, regular and irregular at the origin $r=0$, which are solutions of the radial Schrödinger equation for the pure Coulomb interaction which prevails asymptotically. $E$ is the total energy, $\epsilon_{i}$ are channel energies (i.e., $E=E_{i}^{+}+\epsilon_{i}$ ), and $r_{c}$ is the core boundary. $C_{i, i^{\prime}}$ and $\mathcal{S}_{i, i^{\prime}}$ are the elements of symmetric non-diagonal matrices which account for the short-range scattering $\left(r<r_{c}\right)$. Each radial factor $[. .$.$] in the wavefunction, Eq. (2), is multiplied by the$ appropriate target (ion core) function $\chi_{i}$ and a sum is taken over all channels $i$ in order to construct a general channel function $\Psi_{i^{\prime}}\left(E, q, r_{c}<r\right)$ labeled by a single index $i^{\prime}$. Finally, the most general form of the multichannel wavefunction outside the core is obtained by taking a second sum over all components $i^{\prime}$ with amplitudes $B_{i^{\prime}}$ that account for the channel mixing.

Application of asymptotic boundary conditions to the electron wavefunction components-the closed channel components must vanish asymptotically, whereas in the eigenchannel formulation employed here the open channel components are required to have a common asymptotic phase shift—yields the homogeneous algebraic linear equation system $^{22,23}$

$\sum_{i^{\prime}}\left[\sin \beta_{i}\left(\epsilon_{i}\right) C_{i, i^{\prime}}+\cos \beta_{i}\left(\epsilon_{i}\right) \mathcal{S}_{i, i^{\prime}}\right] B_{i^{\prime}}=0$ for each $i$.

The solution of Eq. (3) for a given total energy $E$ gives the channel mixing coefficients $B_{i^{\prime}}(E)$. Here, the phase parameters $\beta_{i}$ refer to the motion of the Rydberg electron in the pure Coulomb field and derive from the radial Coulomb functions $f$ and $g$, whereas the matrices $\mathcal{S}$ and $C$ are the same as in Eq. (2) and account for the short-range scattering off the $\mathrm{H}_{2}^{+}$ core, which is not included in the phase parameters $\beta_{i}$. The prescriptions of MQDT state that for closed channels (channel energy $\epsilon_{i}<0, i \in Q$ ) the value

$$
\beta_{i}=\pi v_{i}=\pi\left(-\epsilon_{i}\right)^{-1 / 2}
$$

(where $\epsilon_{i}$ is in mass-corrected Rydberg units, $\mathcal{R}_{\mathrm{M}}$ ) must be used, whereas for open channels (channel energy $\epsilon_{i}>0$, $i \in P$ ) it has to be set to the negative of the (as yet unknown) asymptotic electron eigenphase shift $\pi \tau_{\rho}$. In this second situation, Eq. (3) takes the form of a generalized linear algebraic equation system ${ }^{22,23}$ the solution of which yields the phase shifts $\pi \tau_{\rho}$. Note incidentally that the phase relation, Eq. (4), is not unique and corresponds to a specific energy normalization of $f$ and $g$ in the discrete part of the spectrum (the functions called $s$ and $c$ by Seaton). ${ }^{20,24}$ With the alternative choice of Seaton's functions $f$ and $h$ ("Ham"-type normalization), for instance, the $\beta$ parameter deviates from Eq. (4) at low energies and the corresponding quantum defects, usually referred to as " $\eta$ " defects, are also different. ${ }^{25}$ The set of eigenphase shifts $\pi \tau_{\rho}, \rho=1, \ldots, n_{p}$, equals the number $n_{P}$ of open channels and is obtained by solving the linear system, Eq. (3), for any given total energy $E$.

If all channels are closed [or if open channels are arbitrarily removed from Eq. (3)], Eq. (3) can be satisfied only for a discrete set of energies $E$. In this case the effective principal quantum number $n^{*}$, defined by the expression

$$
\begin{aligned}
\frac{\left(n^{*}\right)^{3}}{2}= & \mathcal{N}^{2}=\int_{0}^{\infty} \Psi_{n}^{2} d r \\
= & \frac{1}{\pi} \sum_{i} \frac{\partial \beta_{i}}{\partial E}\left[\sum_{i^{\prime}}\left(\cos \beta_{i} C_{i, i^{\prime}}-\sin \beta_{i} \mathcal{S}_{i, i^{\prime}}\right) B_{i^{\prime}}\right]^{2} \\
& + \text { higher terms, }
\end{aligned}
$$

is a quantity of interest and weighs the contributions of the channels present in the mixed level $n$. The higher terms not given explicitly in Eq. (5) involve the energy derivatives of $C$ and $\mathcal{S}$, see Ref. 22. Note that the single-channel quantity $\partial \beta_{i} / \partial \epsilon_{i}$ has the value $\left(\pi v_{i}^{3}\right) / 2$ owing to the validity of the Rydberg expression $\epsilon_{i}=-1 / v_{i}^{2}$ so that, in the absence of channel mixing $\left(B_{i^{\prime}}=\delta_{i i^{\prime}}, C\right.$, and $\mathcal{S}$ diagonal), Eq. (5) reduces to $n^{*}=v_{i}$. For a single unperturbed Rydberg series $i$ the quantity $\left[2 /\left(n^{*}\right)^{3}\right]$ is simply the level spacing expressed in the unit $\mathcal{R}_{\mathrm{M}}$. The full expression (5) therefore gives the local multichannel level density at $E=E_{n}$ and is identical with the normalization factor $\mathcal{N}^{2}$ that relates the square of the energy-normalized multichannel wavefunction to the unity-normalized wavefunction required in the bound range.

The ratio of the matrices $\mathcal{S}$ and $C$ is $\mathbf{K}$, the reaction matrix of quantum defect theory, which in turn defines the quantum defect matrix, $\boldsymbol{\mu}$,

$$
\sum_{i^{\prime}} \mathcal{S}_{i, i^{\prime}}(E) C_{i^{\prime}, j}^{-1}(E)=K_{i, j}(E) \equiv \tan \left[\pi \mu_{i, j}(E)\right]
$$

(where the $\tan$ is taken for each element individually). Definition Eq. (6) differs from that usually adopted in scattering theory which limits $\mathbf{K}$ to open channels while MQDT may include open as well as closed channels. When only open channels are present, or closed channels are eliminated by application of the condition, Eq. (3), the two definitions coincide.

\section{B. Frame transformation}

The matrix elements $C_{i, i^{\prime}}$ and $\mathcal{S}_{i, i^{\prime}}$ are generated from geometry- and symmetry-dependent clamped-nuclei quantum defect matrices using the rovibrational frame transformation method, which relates a wavefunction expansion expressed in body-frame coordinates for the excited electron to an expansion in terms of lab-frame coordinates appropriate for the electron-ion collision (or half-collision) process of interest. 
In many instances, this transformation is a powerful tool for the description of non-adiabatic dynamics in highly excited molecular states. ${ }^{6,22,23}$ Specifically, if spin effects are neglected and the ion core electronic states may be characterized by their symmetry $\Lambda^{+}$, one has $i=\Lambda^{+}, v^{+}, N^{+}$, where $v^{+}$is the vibrational state and $N^{+}$is the rotational state of the target ion. The frame transformation then takes the form ${ }^{22}$

$$
\begin{aligned}
\mu_{i, i^{\prime}}(E) \equiv & \mu_{\Lambda^{+}, \ell, v^{+}, N^{+} ; \Lambda^{+\prime}, \ell^{\prime}, v^{+\prime}, N^{+\prime}}^{(N p)}(E) \\
= & \sum_{\Lambda}\left\langle N^{+} \mid \Lambda\right\rangle^{\left(N p, \Lambda^{+}, \ell\right)} \\
& \times \int\left[\chi_{v^{+}}^{\left(N^{+}, \Lambda^{+}\right)}(R) \mu_{\Lambda^{+}, \ell ; \Lambda^{+\prime}, \ell^{\prime}}^{(\Lambda)}(\epsilon, R) \chi_{v^{+\prime}}^{\left(N^{+\prime}, \Lambda^{+\prime}\right)}(R)\right] d R \\
& \times\left\langle\Lambda \mid N^{+\prime}\right\rangle^{\left(N p, \Lambda^{+\prime}, \ell^{\prime}\right)} .
\end{aligned}
$$

In this expression, the vibrational transformation is contained in the ion core (target) vibrational wavefunctions $\chi(R)$ whereas the rotational transformation is contained in the matrix elements $\left\langle N^{+} \mid \Lambda\right\rangle$, which are essentially symmetrized vector-coupling coefficients. ${ }^{26}$ The quantum defect matrix elements $\mu_{\Lambda^{+}, \ell ; \Lambda^{+\prime}, \ell^{\prime}}^{(\Lambda)}(\epsilon, R)$ embody the information on the short-range electron-ion scattering process at fixed geometry. The relationship between the body-frame electron energy $\epsilon$ and the total energy $E$ of the vibrating molecule is discussed in Ref. 22. For a given total electronic symmetry $\Lambda$, which is assumed to be conserved at short range, $\mu_{\Lambda^{+}, \ell ; \Lambda^{+}, \ell^{\prime}}^{(\Lambda)}$ connect channels characterized by the electronic symmetry of the core, $\Lambda^{+}$, and the partial wave component of the excited electron, $\ell$. The lab-frame channels are characterized in addition to $\Lambda^{+}$and $\ell$ by the vibrational quantum number $v^{+}$associated with the core state $\Lambda^{+}$and by the total angular momentum $N$ (exclusive of spins) as well as the total parity $p$ (see Ref. 26 for a more complete description and more details).

\section{Determination of quantum defects}

In the present work, as in previous related work, ${ }^{27}$ the electronic quantum defect matrix is effectively reduced to a single element, $\mu^{(\Lambda)}(R)$ for $\Lambda=0$ and 1 , referring to the ion core ground state $X^{+2} \Sigma_{g}^{+}$and corresponding to $\ell=\ell^{\prime}=1$. Consequently, the indices $\Lambda^{+}$and $\ell$ may be dropped in Eq. (7). The two quantum defect functions and their linear and quadratic energy dependences in the range up to threshold have been extracted in Ref. 27 from the best existing ab initio singlet ungerade Born-Oppenheimer potential energy curves ${ }^{28-30}$ by use of the single-closed-channel clamped-nuclei version of the boundary condition, Eq. (3). Note that the adiabatic corrections, which have also been computed for these states in Refs. 28-30, are not required in the MQDT approach. As shown in Ref. 31, they arise in part via the adiabatic corrections of the ion core potential energy curve (see Sec. III F) and in part, along with the non-adiabatic effects, via the nuclear-electronic frame-transformation discussed in Sec. III B. In the quantum defect determination procedure, carried out for $R=1$ to $7 \mathrm{a}_{0}$ on the mesh provided by the $a b$ initio results, it was required that the solutions of the boundary condition exactly reproduce $a b$ initio binding energies for $n=2,3$, and 4 . A specific feature of this determination was that the anticrossing between $\ell, \lambda=1,0$ and 3,0 that occurs for $n=4$ near $R=6$ a a $_{0}$ was diabatized. Another feature, which however is only of limited importance in the present context, is that the bound-state energy normalization was chosen according to the prescription of Ham, ${ }^{20}$ yielding $\eta$ quantum defects. $^{27}$

\section{Photoabsorption and photoionization}

The process of photoabsorption is described in terms of energy-normalized channel transition amplitudes, $D_{i^{\prime}}$, defined as

$$
\begin{aligned}
D_{i^{\prime}}= & \iint \chi_{0}^{*}(q) \psi_{0}^{*}(r) \mathcal{D}(q, r) \\
& \times\left\{\sum_{i} \chi_{i}(q) \frac{1}{r}\left[f_{i}\left(\epsilon_{i}, r\right) C_{i, i^{\prime}}-g_{i}\left(\epsilon_{i}, r\right) \mathcal{S}_{i, i^{\prime}}\right]\right\} \mathrm{d} q \mathrm{~d} r
\end{aligned}
$$

where $\mathcal{D}$ is the transition operator for an electric dipole transition and the ground state is assumed to be represented by a simple product $\Phi_{0}(q, r)=\chi_{0}(q) \psi_{0}(r)$. To the extent that the initial state $\Phi_{0}$ is concentrated near the core or target, the channel transition amplitudes $D_{i^{\prime}}$ may be considered as short-range quantities, with a tendency to vary smoothly with energy - this is indeed the reason for introducing them. Their evaluation, in general, requires the knowledge of $\Psi(E, q, r)$ inside the core, where the superposition coefficients $C_{i, i^{\prime}}$ and $\mathcal{S}_{i, i^{\prime}}$ in the bracket $[\ldots]$ in Eq. (8) deviate from their asymptotic values attained for $r>r_{c}$.

In practice the channel amplitudes $D_{i^{\prime}}$ are obtained by relating them to the clamped-nuclei electronic transition moments $d_{\ell \Lambda \leftarrow X}(\epsilon, R)$ for absorption from the ground state to each excited electronic channel $\ell \Lambda$. We do this here in the way spelled out in detail in Sec. V B of Ref. 26, where the symmetrization and rotational factors involved are also specified. The transformation of the clamped-nuclei electronic transition moments $d_{\ell \Lambda \leftarrow X}(\epsilon, R)$ into asymptotic channel amplitudes $D_{i^{\prime}}$ also involves the frame transformation contained in Eq. (7), see Ref. 26. We have extracted the electronic dipole transition amplitudes $d_{\ell \Lambda \leftarrow X}(\epsilon, R)$ following the method used in Refs. 27 and 32, from the fixed-nuclei $a b$ initio state-to-state transition moments of Wolniewicz and Staszewska, ${ }^{29,30}$ with the difference that here we use $\eta$ instead of $\mu$ quantum defects, so that Seaton' ${ }^{20}$ additional normalization factor $A(E, \ell)$ must be properly included in the conversion from unity-normalized to energy-normalized quantities.

The photoabsorption intensity to an excited state, discrete or continuous, is then described by the superposition of channel amplitudes according to Eq. (8) as

$$
D(E)=\sum_{i^{\prime}}\left[D_{i^{\prime}} B_{i^{\prime}}(E)\right],
$$

where the coefficients $B_{i^{\prime}}$ are those resulting from the solution of the linear equation system, Eq. (3). In the discrete range, the amplitude $D_{n}$ for the transition to a level $n$ at energy $E_{n}$ must be normalized to unity by division through the factor $\mathcal{N}$ from Eq. (5), i.e., $D_{n}=D\left(E_{n}\right) / \mathcal{N}$. In the continuum, partial and total cross sections are evaluated by appropriate superposition of the various eigenchannel functions $\Psi^{(\rho)}(E, q, r)$ obtained 
via application of the boundary conditions, Eq. (3). This is not discussed in detail here; we instead refer to Refs. 22 and 23.

The continuum absorption cross section $\sigma_{a}$ for absorption from a given ground state level $v^{\prime \prime}, N^{\prime \prime}$ is expressed in terms of the total transition dipole moment, Eq. (9), as

$\sigma_{a}(E)=4 \pi^{2} \alpha \frac{1}{\left(2 N^{\prime \prime}+1\right)}\left(E-E_{X, v^{\prime \prime}, N^{\prime \prime}}\right)|D(E)|^{2}$,

where $\alpha$ is the fine-structure constant. The Einstein coefficient $A$ for spontaneous emission relating to an absorption transition from the $X$ ground state toward a bound excited level $n$ is given by

$A_{n \rightarrow X, v^{\prime \prime}, N^{\prime \prime}}=4 \frac{m_{e} c^{2}}{\hbar} \alpha^{5}\left(\frac{E_{n}-E_{X, v^{\prime \prime}, N^{\prime \prime}}}{2 \mathcal{R} h c}\right)^{3}\left(\frac{1}{2 N+1}\right)\left|\frac{D_{n}}{a_{0}}\right|^{2}$,

where $\mathcal{R}$ is the Rydberg constant, and $m_{e}, c, h$, and $a_{0}$ have their usual meanings. As stated above, $D_{n}$ is related to $D$ in Eq. (9) by $D_{n}=D\left(E_{n}\right) / \mathcal{N}$. The factor in front of the parentheses has the numerical value $6.426 \cdot 10^{10} \mathrm{~s}^{-1}$. The energies and transition dipoles are written here in SI units instead of the Rydberg units used in the preceding expressions, but notice that the brackets $(. .$.$) and |\ldots|$ containing $E$ and $D$ actually are dimensionless. The absorption cross section integrated over an individual ionization/dissociation resonance, $n$, may be related, at least approximately, to the line intensity which would be present if there was no interaction between the discrete upper state and the continua. The relationship is

$$
\begin{aligned}
A_{n \rightarrow X, v^{\prime \prime}, N^{\prime \prime}} \approx & 8 \pi c\left(\frac{2 N^{\prime \prime}+1}{2 N+1}\right)\left(\frac{E_{n}-E_{X, v^{\prime \prime}, N^{\prime \prime}}}{h c}\right)^{2} \\
& \times \int_{n} \sigma_{a}(E) d(E / h c)
\end{aligned}
$$

and provides a measure of the intensity of a resonance as a whole. In Eq. (12) $\left(E_{n}-E_{X, v^{\prime \prime}, N^{\prime \prime}}\right) /(h c) \equiv v / c$ is the incident photon wave number and $\int_{n} \sigma_{a}(E) d(E / h c)=\sigma^{(\text {disc })}$ is the cross section integrated over the resonance region.

\section{E. Inclusion of dissociation channels in the MQDT treatment}

Our MQDT approach to the theoretical description of the predissociation of Rydberg states in competition with ionization processes has evolved over the years to become a practical tool for theoretical spectroscopy. ${ }^{22,23,27,33-35}$ An $\mathrm{R}$-matrix type procedure is introduced in order to include dissociation channels along with the ionization channels. In a first step the integration range of the ion core vibrational wavefunctions $\chi(R)$ is limited to a finite rather small value, $R_{c}$, which plays the role of the vibrational core boundary in addition to the electronic core boundary $r_{c}$ which appears in Eq. (2) but actually need not to be specified in the theory. $R_{c}$ is chosen such that all "relevant" bound vibrational Rydberg wavefunctions-represented in terms of the ion-core vibrational functions via expansion Eq. (2)—do not extend out to the boundary and therefore are not affected by this choice. Core vibrational functions with quantum numbers $v^{+}$ far higher than the set of "relevant" states do reach out to the core boundary, however, and they as well as their energies do therefore depend on the choice of $R_{c}$. This is illustrated by Fig. 13 of Ref. 22. This behavior may at first sight appear as unphysical, but in fact it contains the key to our treatment of dissociation channels.

In a second step, we impose a common boundary condition to all vibrational functions at the preselected value of $R=R_{c}$, namely, $b=-\chi^{\prime} /\left.\chi\right|_{R=R_{c}}$, where the prime refers to the derivative with respect to $R$. One consequence of choosing a common boundary condition applied to all core vibrational wavefunctions is that the vibrational continuum is represented for $R \leq R_{c}$ by an orthonormal set of basis functions and is discretized. A further consequence is that via the vibrational frame transformation, Eq. (7), the matrices $C$ and $\mathcal{S}$ also become dependent on $b$ and are logically denoted as $C^{(b)}$ and $\mathcal{S}^{(b)}$. The dependence on $b$ affects matrix elements with indices $i$ and/or $i^{\prime}$ corresponding to high $v^{+}$and consequently of low effective principal quantum number $n^{*}$, essentially $n^{*} \approx 2$ in our application. With the expansion of Eq. (2) chosen to include ion-core vibrational states with conveniently large $v^{+}$ values, quasi-discrete low- $n$ states are obtained when Eq. (3) is solved; see Fig. 3 of Ref. 11 for a graphical illustration. These states have non-vanishing vibrational components at $R=R_{c}$ and therefore connect to the dissociation continua for $R>R_{c}$. These are, in the experiment discussed here, the $2 p \sigma B{ }^{1} \Sigma_{u}^{+}$, $2 p \pi C{ }^{1} \Pi_{u}^{+}$, and $3 p \sigma B^{\prime}{ }^{1} \Sigma_{u}^{+}$states, which all correlate with the $\mathrm{H}(1 s)+\mathrm{H}(\mathrm{n}=2)$ dissociation limit below the energy range studied here. Since the vibrational components for $R<R_{c}$ have a well-defined logarithmic derivative $b$ at the boundary $R_{c}$, they connect for each dissociation channel $d$ to an asymptotic dissociation function characterized by a well-defined dissociation phase-shift.

Based on these ideas a variational R-matrix treatment can be developed which converts the matrices $C^{(b)}$ and $\mathcal{S}^{(b)}$ into "grand" matrices $C$ and $\mathcal{S}$, where the index $b$ may be dropped again, but where the dissociation channels appear explicitly as additional rows and columns in the matrices. This procedure has been initiated in Ref. 34. MQDT expressions (2) and (3) remain unchanged, except that the regular and irregular base-pairs $f_{i}(r)$ and $g_{i}(r)$ must now be identified for $i=d$ with heavy-particle dissociation functions $F_{d}(R)$ and $G_{d}(R)$. At the same time, the target function $\chi_{i}(q)$ in Eq. (2) changes its meaning for $i=d$ : instead of denoting a vibrating ion core along with the partial wave component $\ell$ of the outgoing electron, it now denotes the electronic dissociating molecular state along with the angular part (rotational wavefunction with quantum number $N$ ).

\section{F. Calculations}

In the present application we have $\Lambda^{+}=0$ and $\ell=1$ so that the frame transformation matrix $\left\langle N^{+} \mid \Lambda\right\rangle$ occurring in Eq. (7) has dimension 2 for $N^{c}$ levels (derived from ${ }^{1} \Sigma_{u}^{+}$ and ${ }^{1} \Pi_{u}^{+}$electronic symmetry) and dimension 1 for $N^{d}$ levels (derived from ${ }^{1} \Pi_{u}^{-}$electronic symmetry), cf. Ref. 22 where the explicit expressions are given.

The ion core rovibrational wave functions $\chi_{v^{+}}^{\left(N^{+}, \Lambda^{+}=0\right)}(R)$ required to evaluate the quantum defect matrix elements of Eq. (7) were evaluated in the adiabatic approximation by use of the ion ground state potential energy curve of 
Wind ${ }^{36}$ and the adiabatic correction terms of Bishop and Wetmore. ${ }^{37}$ The adiabatic approximation is not good enough for the corresponding ion levels, which play the role of the ionization limits $v^{+}, N^{+}$of our problem. We have therefore taken them from Wolniewicz and Orlikowski ${ }^{38}$ who evaluated them including the non-adiabatic and relativistic interactions in addition to the adiabatic corrections. These ion level energies, however, do not include the hyperfine interactions, but their accuracy - as well as that of the potential curves and adiabatic corrections-is sufficient in the present context. We have used a box radius of $R_{c}=6 \mathrm{a}_{0}$ and we included 22 vibrational levels $v^{+}$in the ion core vibrational basis, where for $c$ symmetry levels two rotational states per $v^{+}$value must be included for $\ell=1$, namely, $N^{+}=N-1$ and $N^{+}=N+1$, whereas for $d$ symmetry levels a single rotational level must be included for each $v^{+}$value, namely, $N^{+}=N$. We have found that this basis and box radius yield an adequate representation of the bound Rydberg levels in the energy range studied (up to $v=7$ ), as well as a converged representation of the $2 p \pi C^{1} \Pi_{u}^{+}$ and $3 p \sigma B^{\prime 1} \Sigma_{u}^{+}$vibrational continua. At the same time the basis is sufficiently small, so that the continuum associated with the $2 p \sigma B^{1} \Sigma_{u}^{+}$state remains excluded, which we assume to have a negligible effect.

The $X^{1} \Sigma_{g}^{+}$ground state of $\mathrm{H}_{2}$ is well isolated from all singlet excited states so that for the purpose of the intensity calculations we have neglected the non-adiabatic interactions. Its vibrational eigenfunctions and eigenvalues for each rotational quantum number $N^{\prime \prime}$ were evaluated in the adiabatic approximation, adding also the corresponding centrifugal term to the $a b$ initio potential. The adiabatic correction terms and the relativistic and radiative corrections were taken from the work of Wolniewicz. ${ }^{39}$ For the purpose of the calculations of transition energies, we have added the non-adiabatic corrections to the ground-state level energies from Ref. 40. (The most recent theoretical evaluation of the non-adiabatic corrections in $\mathrm{H}_{2}{ }^{41}$ yields a value for the ground level that is $0.002 \mathrm{~cm}^{-1}$ lower than obtained in Ref. 40. This improvement however is not relevant for the present study.)

The photodissociation and photoionization cross section profiles have been evaluated for photon energies from 125600 to $126000 \mathrm{~cm}^{-1}$ on a grid with a spacing of $0.001 \mathrm{~cm}^{-1}$. The calculated profiles include all rotational transitions that correspond to $N^{\prime}, N^{\prime \prime} \leq 3$, namely, $R(0), P(1), Q(1), R(1)$, $P(2), Q(2), R(2), P(3)$, and $Q(3)$. For $Q$ transitions $\left(N^{d}\right.$ upper levels), only the photoionization cross section has been evaluated because dissociation is known to be significant only for a few upper levels with $v^{+}>1$. For $R$ and $P$ transitions ( $N^{c}$ upper levels) on the other hand the full $i+d$ approach outlined in Sec. III E has been used.

Fig. 5 presents an overview of the predicted ionization and dissociation cross sections in the photon range $125600-126000 \mathrm{~cm}^{-1}$. The two cross sections are strikingly different-indeed at first sight it is surprising that they should stem from the same molecule. The dissociation cross section is dominated by characteristic very broad asymmetric resonances, whereas in the ionization spectrum numerous intense sharp peaks stand out. Only a closer look reveals that there are coincidences, corresponding to the minority of

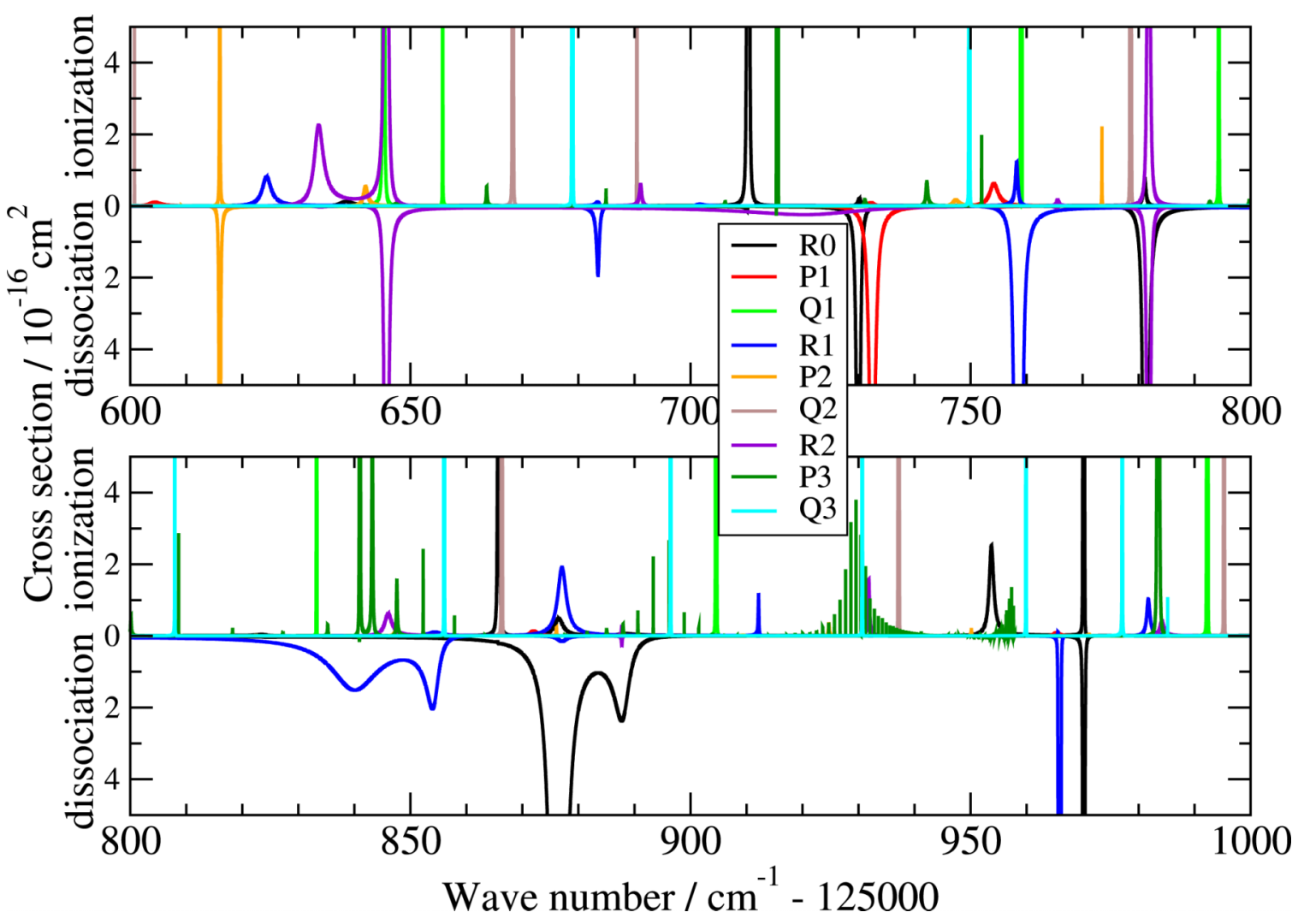

FIG. 5. Calculated ionization (plotted upward) and dissociation (plotted downward) cross sections of $\mathrm{H}_{2}$ for photon energies in the range 125600 and $126000 \mathrm{~cm}^{-1}$. All rotational transitions corresponding to $v^{\prime \prime}=0$ and $N^{\prime}, N^{\prime \prime} \leq 3$ are shown, cf. the color coding indicated in the figure. Ground state rotational population factors have not been included. 
transitions for which the upper levels ionize and dissociate. In the following we use the information contained in Fig. 5 in order to analyze the experimental spectra shown in Fig. 4.

\section{RESULTS AND DISCUSSION}

\section{A. Absorption spectrum}

Fig. 6 presents an overview of the absorption spectrum of $\mathrm{H}_{2}$ recorded in the present work (top) and compares it with the theoretical spectrum calculated by use of the approach outlined in Sec. III (bottom). This latter spectrum is the sum of the dissociation and ionization spectra shown in Fig. 5 (note however the different scales used in Figs. 5 and 6). The present experiment initially yields the transmission signal $\tau_{i}^{\prime}$ (see Sec. II, Fig. 4), but in Fig. 6 we have preferred to plot the quantity $-\ln \tau_{i}^{\prime}$ which corresponds to the product of the absorption cross section $\sigma$ and the effective column density of the experiment.

Our experiment does not give access to the absolute cross section because the column density can only be very roughly estimated. However, Fig. 6 provides the opportunity to calibrate it approximately via a comparison with the theoretical spectrum. This procedure is justified by previous work, ${ }^{32}$ where systematic extended comparisons between MQDT intensity predictions and absolute absorption intensity measurements have yielded agreement experiment/theory to within a factor $1.05 \pm 0.25-$ see, e.g., Fig. 5 of Ref. 42 for a graphical illustration. For the purpose of calibration, the theoretical spectrum in Fig. 6 (bottom) has been convolved with the experimental Doppler width of $0.09 \mathrm{~cm}^{-1}$. This is necessary because most of the intense absorption lines emerging from the background noise are $Q(1)$ transitions that have natural widths far smaller than, or at best comparable to, the Doppler width, cf. Table II in Sec. IV B, and their

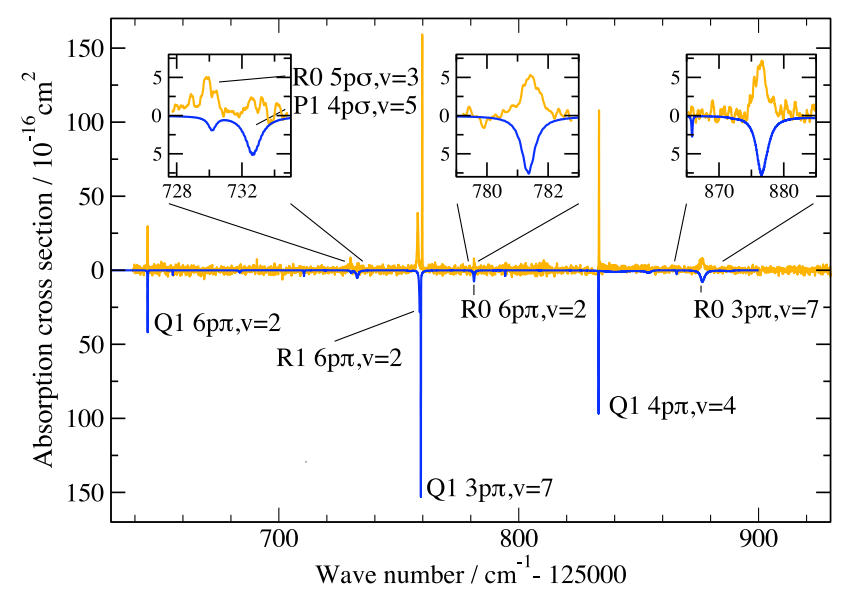

FIG. 6. Absorption spectrum of $\mathrm{H}_{2}$ in the region between the $v^{+}=0$ and $v^{+}$ $=1$ ionization thresholds. Top: experiment (orange), bottom: MDQT, from first principles (blue). The theoretical spectrum has been convolved with a Gaussian line-shape function corresponding to the experimental Doppler width of $0.09 \mathrm{~cm}^{-1}$ (FWHM). The ordinate scale of the upper spectrum has been calibrated by comparison with the theoretical spectrum, cf. the text. Insets: enlarged portions of the spectrum showing the $R(0) 3 p \pi, v=7$ resonance (right), the $R(0) 6 p \pi, v=2$ (center), and the $R(0) 5 p \sigma, v=3$ and $P(1) 4 p \sigma, v=5$ resonances (left). effective peak heights therefore depend sensitively on the exact experimental conditions. Further we have assumed that no spin exchange occurs during the cooling so that the ortho/para ratio corresponds to that at room temperature (ortho/para $=0.75 / 0.25$ ). We have used the two strongest peak maxima for the calibration and found that the effective column density of the present absorption measurements corresponds to about $0.8 \cdot 10^{13}$ molecules $/ \mathrm{cm}^{2}$, in agreement with the estimate $(1.1 \pm 0.4) \cdot 10^{13}$ molecules $/ \mathrm{cm}^{2}$ made in Sec. II. Fig. 6 shows overall reasonable agreement as far as the weaker sharp peaks are concerned. $R(0) 6 p \pi, v=2$, for instance, is quite accurately reproduced whereas the associated $Q(1)$ and $R(1)$ transitions agree to within about $30 \%$.

A few much broader peaks are also visible in Fig. 6, such as the $3 p \pi, v=7 R(0)$ peak which has a width of nearly $2 \mathrm{~cm}^{-1}$ and is not affected by the convolution procedure. The fact that its calculated peak maximum matches the calibrated experimental value (cf. the inset on the right) is satisfying and confirms the internal consistency of the calibration procedure. The same is true-although the agreement is less quantitative here-for the $R(0) 6 p \pi, v=2$ peak, which has a calculated width of $0.50 \mathrm{~cm}^{-1}$ (inset in the center of the figure). Finally, the $R(0) 5 p \sigma, v=3$ and $P(1) 4 p \sigma, v=5$ peaks near $125730 \mathrm{~cm}^{-1}$ are just barely visible in the absorption spectrum of Fig. 6. They have calculated widths of 0.55 and $1.09 \mathrm{~cm}^{-1}$, respectively, again much larger than the Doppler width. These two peaks are represented on an enlarged scale in the inset on the left of Fig. 6. Here we have taken a running average over the experimental points in order to smooth out the noise. The agreement between the theoretical spectrum and the calibrated experimental spectrum is less striking here, but may still be considered satisfactory.

Overall, the absorption spectrum of Fig. 6 is striking as only few transitions clearly emerge from the background noise. Nearly all of these are sharp $Q(1)$ transitions with natural widths smaller than the Doppler broadening of $0.09 \mathrm{~cm}^{-1}$ (cf. Table II in Subsection IV B). Note that, for instance, the $3 p \pi, v=7 Q(1)$ and $R(0)$ transitions have comparable overall intensity as indicated by their Einstein $A$ coefficients $\left(24 \cdot 10^{6} \mathrm{~s}^{-1}\right.$ and $28 \cdot 10^{6} \mathrm{~s}^{-1}$, respectively, last column of Table II). Due to its large width of nearly $2 \mathrm{~cm}^{-1}$, the latter has a rather low peak maximum intensity, which is nearly buried in the noise, whereas the high peak maximum intensity of the former results from the combined effects of its small intrinsic width, the small Doppler broadening, and the high spectral resolution achieved in the present experiment.

\section{B. Photoionization spectrum}

\section{Intensity calibration}

An overview of the experimental photoionization spectrum obtained in this work is presented in the upper panel of Fig. 4. The photoionization spectrum exhibits less noise than the absorption spectrum and shows a much richer structure. As in the case of the photoabsorption spectrum, we have calibrated it by comparison with the spectrum obtained theoretically. To this end, we have convoluted the theoretical spectrum with the experimental Doppler width of $0.04 \mathrm{~cm}^{-1}$. 
A preliminary inspection of the observed and calculated spectra revealed that transitions corresponding to $N^{\prime \prime}=2$ and 3 could be clearly identified and appear with surprisingly strong intensities, indicating that the $\mathrm{H}_{2}$ gas sample is only partially cooled. According to the discussion given in Sec. II this is due to the contribution from the room-temperature background gas in the photoionization chamber which has not been subtracted (unlike for the photoabsorption spectrum). We determined the rotational distribution as follows: (i) We assumed that the ortho/para ratio corresponds to that expected for a room temperature sample $(75 \% / 25 \%)$ and (ii) we determined the $N^{\prime \prime}=2 / N^{\prime \prime}=0$ and $N^{\prime \prime}=3 / N^{\prime \prime}=1$ ratios on the basis of selected transitions. The procedure and results are documented by Table I. The rotational distribution given in Table I is in excellent agreement with the estimate made in the experimental Sec. II. The overall calibration of the experimental spectrum is based on selected $v=1$ peaks which are fully ionized according to the calculations. We used several $R(0), P(1), Q(1)$, and $R(1)$ resonance peak maxima for the calibration, and we have taken the mean value of the adjustment factors obtained for the various peaks.

Figs. 7-10 display the final results, which are analyzed in Subsection IV B 2. In the upper panel of each figure, a section of the convoluted calculated spectrum is compared with the calibrated observed spectrum. The lower panel presents the various calculated unconvoluted transitions separately, which are plotted using different colors. The comparison between experiment and theory is gratifying, both in terms of the positions of the peaks and their shapes. The presence of numerous very sharp peaks in the observed and calculated spectra is the result of the high effective resolution attained here and underlines the fact that the broader structures represent the genuine variations of the continuum cross section, that is, its true shape.

\section{Analysis}

The observed photoionization spectrum has been analyzed by comparing the calculated and observed peaks visible in Figs. 7-10, and with reference to the information contained in the lower panel of each figure. We also used information obtained from a calculation of the hypothetical discretelevel spectrum for each type of transition, in which all open channels were artificially removed from the multichannel expansion, Eq. (2). Table II lists the observed and calculated peaks and their assignments thus obtained for $N^{\prime \prime}=0$ and 1 , while Table III contains the corresponding information for the peaks assigned to transitions from $N^{\prime \prime}=2$ and 3. Unless indicated otherwise, the data collected in the various columns of the tables have been extracted from the photoionization spectrum.

- Column 1. The assignments given in the first column of each table correspond to the largest component in the multichannel expansion, Eq. (2) (largest coefficient $B_{i^{\prime}}$ ), restricted to closed channels. Note that these assignments are only indications and useful for bookkeeping purposes, as they do not take account of the vibronic mixing which in some cases is very strong. For instance, the $R(0)$ transitions to $4 p \sigma, v=5$ and $5 p \sigma, v=3$ were assigned in Ref. 2 to the resonances observed then at 125730.3 and $125885.2 \mathrm{~cm}^{-1}$, respectively, whereas here we invert the assignments on the basis of the criteria just mentioned. In reality, however, the two upper levels are calculated to be nearly 1:1 (antisymmetric and symmetric) mixtures of the $v^{+}=3$ and $v^{+}=5$ channels with significant admixture of $3 p \pi, v=7$ and various additional minor contributions. In Table II, the assignments for such strongly mixed levels, for which no single channel contribution exceeds about 30\%, are highlighted by quotation marks “...".

- Columns 2 and 3. In a multichannel situation where resonances are not always well isolated and often overlapping, the concepts of resonance position and width are no longer sharply defined and may depend on how the experimental cross sections are interpreted and how the spectrum is calculated. We have therefore chosen to list in Tables II and III the experimental and theoretical positions of the intensity maxima, which are well defined. The tables show that the observed energies of the peak maxima are fairly well reproduced by the MQDT calculations, e.g., with a mean observed-calculated deviation of $-0.18 \mathrm{~cm}^{-1}$ for 31 measured $N^{\prime \prime}=0$ and 1 transitions, and only a few values corresponding to low $n^{*}$ values exceeding $1 \mathrm{~cm}^{-1}$. The mean value observed-calculated for 46 measured transitions with $N^{\prime \prime}=2$ and 3 is $-0.13 \mathrm{~cm}^{-1}$. The larger deviations are, with only two exceptions, all negative, that is, the predicted energies are too high, probably because of a slight lack of convergence of the $a b$ initio potential energy curves which we use here. The mean value of the absolute values observedcalculated amounts to $0.31 \mathrm{~cm}^{-1}$ for $N^{\prime \prime}=0$ and 1 and to $0.17 \mathrm{~cm}^{-1}$ for $N^{\prime \prime}=2$ and 3 .

- Column 4. The effective principal quantum number $n^{*}$, evaluated according to Eq. (5) which disregards open

TABLE I. Relative ground state rotational populations estimated from comparison with the experimental photoionization spectrum.

\begin{tabular}{cccc}
\hline \hline Rotational level & $n_{N^{\prime \prime}}(\%)$ Room temperature & $n_{N^{\prime \prime}}(\%)$ Present & Determination \\
\hline$N^{\prime \prime}=0$ & 14.0 & 17.7 & \\
2 & 10.8 & 7.4 & $4 p \pi, v=4 R(0)$ and $P(2)$ \\
\hline$N^{\prime \prime}=1$ & 67.7 & 70.8 & \\
3 & 7.2 & 4.1 & $14-18 p 3, v=1 Q(1)$ and $Q(3)$ \\
\hline \hline
\end{tabular}




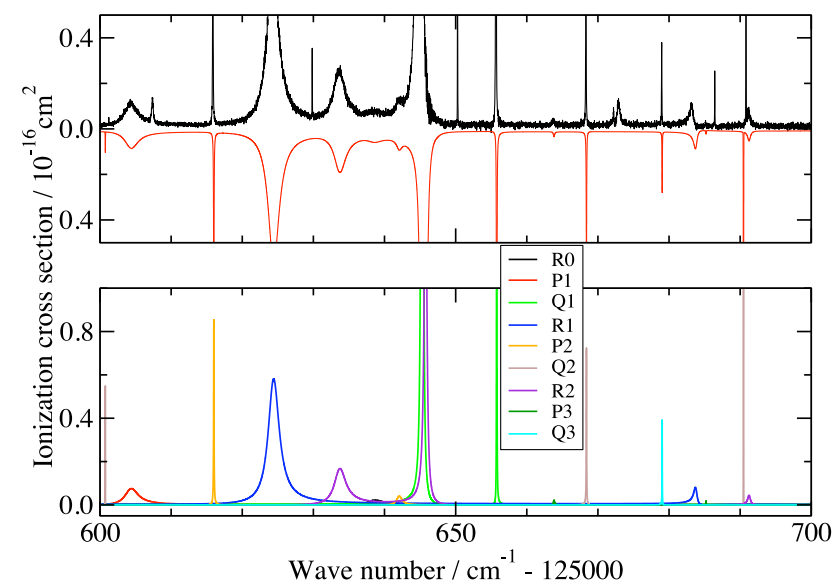

FIG. 7. Photoionization spectrum of $\mathrm{H}_{2}$ in the region between the $v^{+}=0$ and $v^{+}=1$ ionization thresholds: $125600-125700 \mathrm{~cm}^{-1}$. Upper panel: experiment (black) and theory (red). The theoretical spectrum represents the sum of the contributions $N^{\prime \prime}, N \leq 3$ from the lower panel, convolved with the experimental Doppler width of $0.04 \mathrm{~cm}^{-1}$. Lower panel: MDQT, from first principles. The individual transitions are color coded as indicated in the figure and are weighted according to the ground state populations given in Table I.

channels, is often helpful to establish an assignment for a resonance.

- Columns 5 and 6. Just as the resonance positions, the resonance widths are strictly defined only in situations of isolated resonances. Here we proceed in a pragmatic fashion in order to extract widths (FWHM) from our data. The theoretical resonance widths have been obtained from the energy-derivative of the eigenphase sum, i.e., the sum of the phase shifts $\pi \tau_{\rho}$ resulting from the solution of the generalized algebraic linear system, Eq. (3), whereby the sum runs over all open ionization and dissociation channels. For an isolated resonance the energy derivative of the eigenphase sum yields a Lorentzian shape whose maximum is related to the width by

$$
\left[\frac{\partial}{\partial E}\left(\sum_{\rho} \pi \tau_{\rho}(E)\right)\right]_{\max }=\frac{2}{\Gamma}
$$

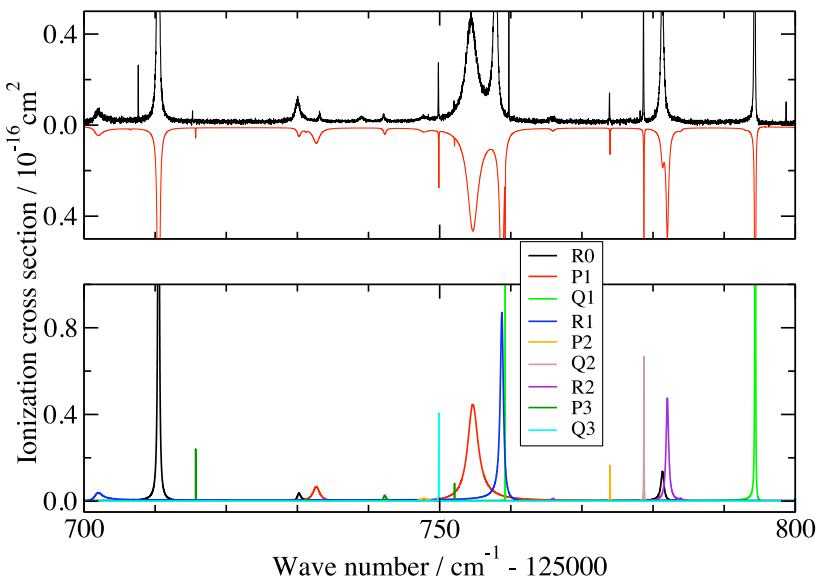

FIG. 8. Photoionization spectrum of $\mathrm{H}_{2}$ in the region between the $v^{+}=0$ and $v^{+}=1$ ionization thresholds: $125700-125800 \mathrm{~cm}^{-1}$, cf. the caption of Fig. 7.

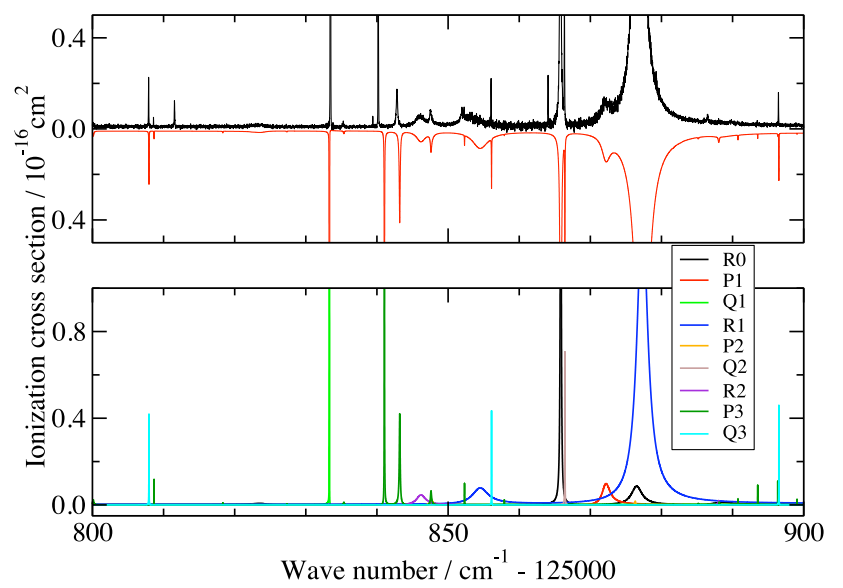

FIG. 9. Photoionization spectrum of $\mathrm{H}_{2}$ in the region between the $v^{+}=0$ and $v^{+}=1$ ionization thresholds: $125800-125900 \mathrm{~cm}^{-1}$, cf. the caption of Fig. 7 .

In our calculations, we have identified the maxima corresponding to the left-hand-side of Eq. (13) and equated their values to the right-hand-side of the same expression. Thereby any effects due to overlapping of resonances are obviously ignored. The experimental resonance widths have been taken from the FWHM of the cross section for each resonance. In a few cases a Beutler-Fano analysis has been carried out, but in many cases the width was obtained essentially by eye from the uppermost plot of each of Figs. 7-10. In the case of narrow resonances that are hardly larger than the Doppler width, we have accounted for the latter by means of the relation $\Gamma=\left[\Gamma_{\text {obs }}^{2}-\Gamma_{\text {Doppler }}^{2}\right]^{1 / 2}$ (which strictly applies only when the natural line shape is Gaussian). Obviously, this pragmatic way of estimating the resonance widths disregards any influence of the asymmetry of the resonances. Columns 5 and 6 of Tables II and III show that, nevertheless, the observed and calculated widths are in quite good agreement despite the fact that they are not well defined quantities.

- Columns 7 and 8. The observed and calculated cross section maxima $\sigma_{i}^{(\max )}$ are well-defined and are given in

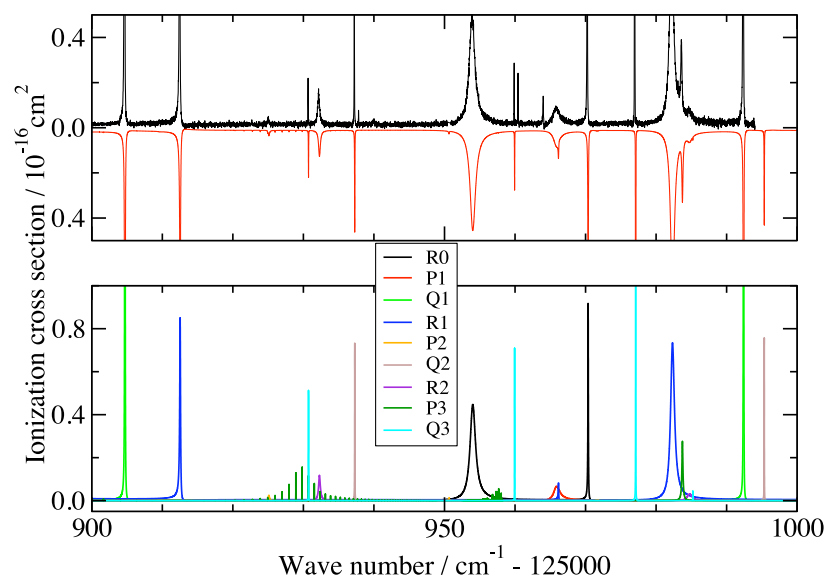

FIG. 10. Photoionization spectrum of $\mathrm{H}_{2}$ in the region between the $v^{+}=0$ and $v^{+}=1$ ionization thresholds: $125900-126000 \mathrm{~cm}^{-1}$, cf. the caption of Fig. 7. 
TABLE II. Photoionization transitions in $\mathrm{H}_{2}$ with $N^{\prime \prime}=0$ and 1. $v / c$, transition wave number. $n^{*}$, effective quantum number/normalization factor [Eq. (5)]. $\Gamma / h c$, resonance width (FWHM). $\sigma_{i}^{(\max )}$, ionization cross section at peak maximum. $\sigma_{i} / \sigma_{a}$, ionization yield at peak maximum. A, Einstein coefficient for spontaneous emission corresponding to the absorption transition to the discrete upper level. n.o. means not observed and the corresponding values indicated in the second column are the calculated ones. Cal. means calculated. Obs. means observed.

\begin{tabular}{|c|c|c|c|c|c|c|c|c|c|c|}
\hline \multirow[b]{2}{*}{ Assignment } & \multicolumn{2}{|c|}{$\frac{v}{c}\left(\mathrm{~cm}^{-1}\right)$} & \multirow{2}{*}{$\begin{array}{c}n^{*} \\
\text { Calc. }\end{array}$} & \multicolumn{2}{|c|}{$\frac{\Gamma}{h c}\left(\mathrm{~cm}^{-1}\right)$} & \multicolumn{2}{|c|}{$\sigma_{i}^{(\max )}\left(10^{-16} \mathrm{~cm}^{2}\right)$} & \multirow{2}{*}{$\begin{array}{c}\frac{\sigma_{i}}{\sigma_{a}}(\%) \\
\text { Calc. }\end{array}$} & \multicolumn{2}{|c|}{$A\left(10^{6} \mathrm{~s}^{-1}\right)$} \\
\hline & Obs. & Obs.-calc. & & Obs. & Calc. & Obs. & Calc. & & Obs. & Calc. \\
\hline$R(0) 10 p 2, v=1$ & 125638.50 & -0.06 & 9.36 & $\approx 1.6$ & 2.48 & 0.078 & 0.065 & 97 & $\approx 0.45$ & 0.30 \\
\hline $11 p 0, v=1$ & 710.42 & -0.06 & 10.50 & 0.22 & 0.20 & 3.14 & 2.75 & 99 & 2.46 & 2.12 \\
\hline$" 5 p \sigma, v=3 "$ & 730.04 & -0.18 & 4.36 & 0.81 & 0.55 & 0.10 & 0.06 & 3 & 9.53 & 3.00 \\
\hline$" 6 p \pi, v=2 "$ & 781.31 & n.o. & 4.66 & $\ldots$ & 0.50 & $\ldots$ & 0.19 & 3 & $\ldots$ & 7.98 \\
\hline $12 p 0, v=1$ & 823.46 & -0.03 & 8.77 & $\approx 1.5$ & 2.04 & 0.018 & 0.016 & 53 & $\approx 0.18$ & 0.0002 \\
\hline $11 p 1, v=1$ & 865.78 & -0.06 & 10.87 & 0.17 & 0.15 & 1.99 & 2.19 & 98 & 1.21 & 1.09 \\
\hline $3 p \pi, v=7^{\mathrm{a}}$ & 876.28 & -0.27 & 3.37 & 2.3 & 1.77 & 7.1 & 7.3 & 2 & 57.64 & 28.09 \\
\hline$" 4 p \sigma, v=5 "$ & 889.7 & 1.1 & 3.63 & $\approx 1.8$ & 3.33 & $\approx 0.03$ & 0.012 & 4 & $\approx 4.8$ & 1.57 \\
\hline $13 p 0, v=1$ & 953.91 & -0.09 & 11.60 & 0.92 & 0.93 & 0.50 & 0.45 & 100 & 1.62 & 1.68 \\
\hline $4 p \pi, v=4$ & 970.23 & -0.14 & 4.00 & 0.09 & 0.07 & 1.11 & 0.79 & 13 & 2.71 & 2.99 \\
\hline $12 p 2, v=1$ & 996.52 & n.o. & 11.97 & $\ldots$ & 0.88 & $\ldots$ & $\ldots$ & 47 & $\ldots$ & 0.0018 \\
\hline$P(1) 11 p 1, v=1$ & 125604.30 & -0.11 & 6.26 & 2.27 & 2.25 & 0.12 & 0.09 & 88 & 2.45 & 2.97 \\
\hline $4 p \sigma, v=5^{\mathrm{a}}$ & 732.87 & 0.22 & 3.96 & $\approx 1.1$ & 1.09 & $\approx 2.8$ & 5.1 & 1 & $\approx 24.4$ & 25.80 \\
\hline $12 p 1, v=1$ & 754.48 & -0.18 & 9.36 & 1.98 & 1.78 & 0.47 & 0.47 & 94 & 7.85 & 5.82 \\
\hline $13 p 1, v=1$ & 872.23 & 0.00 & 12.46 & 2.22 & 1.35 & 0.12 & 0.15 & 98 & 2.16 & 1.40 \\
\hline $14 p 1, v=1$ & 965.83 & -0.10 & 13.39 & 1.16 & 1.05 & 0.09 & 0.09 & 96 & 0.86 & 0.82 \\
\hline$Q(1) 6 p \pi, v=2$ & 125645.25 & -0.05 & 6.68 & $\ldots$ & 0.067 & sat. & 57.40 & 100 & $\ldots$ & 11.86 \\
\hline $11 p 1, v=1$ & 655.66 & -0.11 & 8.19 & $0.038^{\mathrm{b}}$ & 0.026 & 5.61 & 5.55 & 100 & 0.56 & 0.66 \\
\hline $3 p \pi, v=7$ & 759.73 & 0.52 & 3.09 & $0.00^{\mathrm{b}}$ & $0.002^{\mathrm{c}}$ & 0.83 & 1.10 & $0.3^{\mathrm{c}}$ & $\ldots$ & 24.16 \\
\hline $12 p 1, v=1$ & 794.28 & -0.09 & 12.05 & $0.074^{\mathrm{b}}$ & 0.055 & 4.39 & 5.16 & 100 & 0.85 & 0.97 \\
\hline $4 p \pi, v=4$ & 833.46 & 0.15 & 4.06 & $0.04^{\mathrm{b}}$ & $0.001^{\mathrm{c}}$ & sat. & 24.54 & $12^{\mathrm{c}}$ & $\ldots$ & 16.31 \\
\hline $13 p 1, v=1$ & 904.63 & -0.07 & 13.07 & $0.065^{\mathrm{b}}$ & 0.045 & 3.92 & 4.68 & 100 & 0.67 & 0.76 \\
\hline $14 p 1, v=1$ & 992.34 & -0.06 & 14.07 & $0.049^{b}$ & 0.036 & 4.67 & 4.58 & 100 & 0.60 & 0.65 \\
\hline$R(1) 11 p 1, v=1$ & 125624.29 & -0.13 & 10.17 & 1.90 & 1.86 & 0.75 & 0.60 & 99 & 2.28 & 2.03 \\
\hline$" 4 p \sigma, v=5 "$ & 683.17 & -0.54 & 4.28 & 0.77 & 0.57 & 0.10 & 0.09 & 6 & 2.03 & 1.68 \\
\hline $10 p 3, v=1$ & 702.01 & 0.00 & 8.45 & 1.34 & 1.23 & 0.06 & 0.05 & 87 & 0.15 & 0.18 \\
\hline$" 6 p \pi, v=2 "$ & 757.87 & -0.88 & 4.29 & 0.58 & 0.52 & 0.96 & 0.90 & 3 & 29.45 & 18.65 \\
\hline $12 p 1, v=1$ & 783.62 & -0.20 & 9.48 & $\approx 0.4$ & 0.53 & $\approx 0.02$ & 0.03 & 27 & $\approx 0.05$ & 0.0051 \\
\hline $3 p \pi, v=7$ & 840.23 & n.o. & 3.30 & $\ldots$ & 10.14 & $\ldots$ & 0.00 & 0 & $\ldots$ & 17.54 \\
\hline$" 5 p \sigma, v=3 "$ & 853.03 & -1.50 & 4.16 & $\approx 2.8$ & 2.55 & 0.06 & 0.08 & 5 & $\approx 5.3$ & 1.54 \\
\hline $13 p 1, v=1$ & 876.90 & -0.39 & 7.55 & 2.08 & 1.80 & 1.22 & 1.42 & 92 & 4.38 & 3.94 \\
\hline $11 p 3, v=1$ & 912.44 & -0.08 & 11.90 & 0.16 & 0.14 & 0.95 & 0.81 & 99 & 0.24 & 0.17 \\
\hline $4 p \pi, v=4$ & 964.00 & -2.16 & 3.97 & 0.10 & 0.08 & 0.14 & 0.13 & 2 & 1.11 & 1.50 \\
\hline $14 p 1, v=1$ & 982.20 & -0.12 & 12.57 & 0.65 & 0.65 & 0.89 & 0.74 & 99 & 0.92 & 0.82 \\
\hline Mean deviation & & -0.18 & & & & & & & & \\
\hline
\end{tabular}

a Observed only in absorption. The cross section values given refer to absorption.

${ }^{\mathrm{b}}$ Evaluated according to $\Gamma=\left[\Gamma_{\text {obs }}^{2}-\Gamma_{\text {res }}^{2}\right]^{1 / 2}$ where $\Gamma_{\text {res }}=0.04 \mathrm{~cm}^{-1}$ is the effective resolution width

${ }^{c}$ From Ref. 11. These levels have been shown to decay primarily by molecular fluorescence.

columns 7 and 8 of Tables II and III. They corroborate, by and large, the overall calibration discussed in Sec. IV B 1. As may be assessed also from Figs. 7-10, the agreement is less quantitative than for the transition frequencies.

- Column 9 (third to last column) of Tables II and III lists the theoretical quantum yield $\sigma_{i} / \sigma_{a}$ for photoionization. This quantity has been evaluated at the cross section maximum for each resonance from the partial ionization and dissociation cross sections and is a priori distinct from the quantum yield integrated over a given resonance. The yield values given in Tables II and III have been obtained directly from the theoretical cross sections plotted in Fig. 5.
- Column 10 (second to last column). A rough experimental estimate of the peak-integrated intensity [Einstein A coefficient, cf. relation (12)] may be obtained from the observed widths and peak heights. For a Lorentzian resonance the simple relation $\sigma^{(d i s c)}=\left[\pi \Gamma \sigma^{(\max )}\right] / 2$ holds, so that expression (12) takes the form

$$
\begin{aligned}
A_{n \rightarrow X, v^{\prime \prime}, N^{\prime \prime}} \approx & 4 \pi^{2} c\left(\frac{2 N^{\prime \prime}+1}{2 N+1}\right)\left(\frac{v}{c}\right)^{2} \Gamma \sigma_{i}^{(\max )} \\
& \times\left[\frac{\sigma_{a}^{(\max )}}{\sigma_{i}^{(\max )}}\right] \frac{1}{n_{N^{\prime \prime}}} .
\end{aligned}
$$

Here we have, for the purpose of practical application to the photoionization spectrum at hand, divided by the 
TABLE III. Photoionization transitions in $\mathrm{H}_{2}$ with $N^{\prime \prime}=2$ and 3. $v / c$, transition wave number. $n^{*}$, effective quantum number/normalization factor [Eq. (5)]. $\Gamma / h c$, resonance width (FWHM). $\sigma_{i}^{(\max )}$, ionization cross section at peak maximum. $\sigma_{i} / \sigma_{a}$, ionization yield at peak maximum. A, Einstein coefficient for spontaneous emission corresponding to the absorption transition to the discrete upper level. n.o. means not observed and the corresponding values indicated in the second column are calculated ones. Calc. means calculated. Obs. means observed.

\begin{tabular}{|c|c|c|c|c|c|c|c|c|c|c|}
\hline \multirow[b]{2}{*}{ Assignment } & \multicolumn{2}{|c|}{$\frac{v}{c}\left(\mathrm{~cm}^{-1}\right)$} & \multirow{2}{*}{$\begin{array}{c}n^{*} \\
\text { Calc. }\end{array}$} & \multicolumn{2}{|c|}{$\frac{\Gamma}{h c}\left(\mathrm{~cm}^{-1}\right)$} & \multicolumn{2}{|c|}{$\sigma_{i}^{(\max )}\left(10^{-16} \mathrm{~cm}^{2}\right)$} & \multirow{2}{*}{$\begin{array}{c}\frac{\sigma_{i}}{\sigma_{a}}(\%) \\
\text { Calc. }\end{array}$} & \multicolumn{2}{|c|}{$A\left(10^{6} \mathrm{~s}^{-1}\right)$} \\
\hline & Obs. & Obs.-calc. & & Obs. & Calc. & Obs. & Calc. & & Obs. & Calc. \\
\hline$P(2) 4 p \pi, v=4$ & 125615.86 & -0.14 & 3.99 & $0.08^{\mathrm{a}}$ & 0.07 & 1.02 & 0.76 & 13 & 26.48 & 18.05 \\
\hline $12 p 2, v=1$ & 642.11 & +0.01 & 11.97 & $\approx 0.7$ & 0.83 & 0.14 & 0.10 & 98 & $\approx 4.22$ & 1.44 \\
\hline $13 p 0, v=1$ & 695.50 & n.o. & 13.22 & $\ldots$ & 0.27 & $\ldots$ & 0.0099 & 60 & $\ldots$ & 0.0019 \\
\hline $13 p 2, v=1$ & 747.73 & -0.10 & 13.04 & $\approx 0.8$ & 1.09 & 0.037 & 0.031 & 97 & $\approx 1.29$ & 0.62 \\
\hline $14 p 0, v=1$ & 773.88 & -0.08 & 14.32 & $0.06^{\mathrm{a}}$ & 0.04 & 0.14 & 0.13 & 100 & 0.35 & 0.27 \\
\hline $15 p 0, v=1$ & 823.38 & -0.12 & 15.01 & $\approx 0.9$ & 0.50 & 0.019 & 0.016 & 75 & $\approx 0.96$ & 0.018 \\
\hline $14 p 2, v=1$ & 847.70 & n.o. & 14.04 & $\ldots$ & 0.56 & $\ldots$ & 0.020 & 98 & $\ldots$ & 0.45 \\
\hline $16 p 0, v=1$ & 876.32 & n.o. & 16.41 & $\ldots$ & 0.08 & $\ldots$ & 0.017 & 99 & $\ldots$ & 0.061 \\
\hline $17 p 0, v=1$ & 909.65 & n.o. & 15.17 & $\ldots$ & 0.55 & $\ldots$ & 0.0016 & 85 & $\ldots$ & 0.034 \\
\hline $15 p 2, v=1$ & 925.04 & -0.06 & 15.33 & $\approx 0.2$ & 0.18 & 0.042 & 0.037 & 100 & $\approx 0.35$ & 0.20 \\
\hline $18 p 0, v=1$ & 950.64 & n.o. & 17.77 & $\ldots$ & 0.10 & $\ldots$ & 0.028 & 100 & $\ldots$ & 0.054 \\
\hline $7 p \sigma, v=2$ & 971.51 & -0.17 & 11.78 & $\approx 0.2$ & 0.31 & 0.023 & 0.015 & 93 & $\approx 0.21$ & 0.038 \\
\hline $16 p 2, v=1$ & 984.61 & n.o. & 13.48 & $\ldots$ & 0.02 & $\ldots$ & 0.0026 & 93 & $\ldots$ & 0.0019 \\
\hline$Q(2) 3 p \pi, v=7$ & 125601.24 & 0.49 & 3.09 & $\leq 0.03$ & $0.002^{b}$ & 0.05 & 0.10 & $0.1^{\mathrm{b}}$ & $\ldots$ & 22.99 \\
\hline $12 p 2, v=1$ & 668.32 & -0.07 & 12.04 & $0.072^{\mathrm{a}}$ & 0.055 & 0.75 & 0.60 & 100 & 1.37 & 1.04 \\
\hline $4 p \pi, v=4$ & 690.82 & +0.35 & 4.07 & $0.00^{\mathrm{a}}$ & $0.001^{b}$ & 2.14 & 2.68 & $7^{\mathrm{b}}$ & $\ldots$ & 17.00 \\
\hline $13 p 2, v=1$ & 778.66 & -0.06 & 13.07 & $0.062^{\mathrm{a}}$ & 0.045 & 0.55 & 0.51 & 100 & 0.86 & 0.77 \\
\hline $14 p 2, v=1$ & 866.35 & -0.07 & 14.07 & $0.057^{\mathrm{a}}$ & 0.036 & 0.53 & 0.53 & 100 & 0.76 & 0.65 \\
\hline $15 p 2, v=1$ & 937.21 & -0.07 & 15.07 & $\leq 0.042^{\mathrm{a}}$ & 0.030 & 0.53 & 0.47 & 100 & 0.56 & 0.55 \\
\hline$R(2) 9 p 2, v=1$ & 125633.62 & -0.12 & 8.50 & 2.1 & 1.96 & 0.27 & 0.19 & 99 & 10.35 & 5.69 \\
\hline $4 p \sigma, v=5$ & 644.58 & -1.17 & 4.29 & 0.41 & 0.52 & 2.01 & 2.16 & 50 & 29.80 & 28.03 \\
\hline $10 p 4, v=1$ & 691.20 & -0.06 & 10.45 & 0.46 & 0.54 & 0.084 & 0.054 & 88 & 0.79 & 0.37 \\
\hline $3 p \pi, v=7$ & 720.61 & n.o. & 3.07 & $\ldots$ & 23.53 & $\ldots$ & 0.00 & 0 & $\ldots$ & 5.40 \\
\hline $13 p 2, v=1$ & 765.86 & -0.08 & 8.30 & $\approx 0.7$ & 0.49 & 0.033 & 0.027 & 77 & $\approx 0.54$ & 0.12 \\
\hline $5 p \sigma, v=3$ & 781.29 & -0.72 & 5.52 & 0.45 & 0.42 & 0.68 & 0.49 & 43 & 12.87 & 8.48 \\
\hline $14 p 2, v=1$ & 846.11 & -0.10 & 11.02 & $\approx 1.2$ & 1.51 & 0.059 & 0.058 & 100 & $\approx 1.28$ & 1.25 \\
\hline $11 p 4, v=1$ & 882.74 & n.o. & 8.99 & $\ldots$ & 0.40 & $\ldots$ & 0.0017 & 82 & $\ldots$ & 0.0051 \\
\hline $4 p \pi, v=4$ & 888.02 & -0.04 & 4.03 & $\approx 0.2$ & 0.12 & 0.039 & 0.060 & 48 & $\approx 0.29$ & 0.098 \\
\hline $15 p 2, v=1$ & 932.15 & -0.13 & 14.05 & 0.34 & 0.29 & 0.15 & 0.13 & 99 & 0.93 & 0.62 \\
\hline $12 p 4, v=1$ & 984.67 & -0.03 & 14.40 & $\approx 0.5$ & 0.80 & 0.082 & 0.067 & 98 & $\approx 0.76$ & 0.45 \\
\hline$P(3) 14 p 3, v=1$ & 125663.73 & -0.10 & 14.58 & $\approx 0.2$ & 0.19 & 0.045 & 0.035 & 99 & $\approx 0.58$ & 0.28 \\
\hline $20 p 1, v=1$ & 685.14 & -0.07 & 18.60 & $\approx 0.1$ & 0.053 & 0.035 & 0.023 & 99 & $\approx 0.23$ & 0.069 \\
\hline $21 p 1, v=1$ & 706.55 & n.o. & 12.05 & $\ldots$ & 0.16 & $\ldots$ & 0.022 & 97 & $\ldots$ & 0.048 \\
\hline $7 p 1, v=2$ & 715.25 & -0.45 & 8.36 & $\leq 0.03$ & 0.007 & 0.063 & 0.058 & 96 & 0.13 & 0.080 \\
\hline $21 p 1, v=1$ & 731.19 & n.o. & 12.01 & $\ldots$ & 0.24 & $\ldots$ & 0.032 & 98 & $\ldots$ & 0.11 \\
\hline $15 p 3, v=1$ & 742.13 & -0.16 & 11.11 & $\approx 0.3$ & 0.39 & 0.046 & 0.042 & 100 & $\approx 0.50$ & 0.67 \\
\hline $23 p 1, v=1$ & 752.01 & -0.08 & 20.49 & $\leq 0.02$ & 0.018 & 0.11 & 0.09 & 100 & 0.141 & 0.093 \\
\hline $16 p 3, v=1$ & 800.08 & -0.04 & 18.22 & $\approx 0.2$ & 0.15 & 0.027 & 0.033 & 100 & $\approx 0.35$ & 0.23 \\
\hline $27 p 1, v=1$ & 808.58 & -0.08 & 23.74 & $\leq 0.01$ & 0.010 & 0.051 & 0.044 & 100 & 0.033 & 0.073 \\
\hline $30 p 1, v=1$ & 835.27 & -0.09 & 15.04 & 0.13 & 0.13 & 0.035 & 0.022 & 100 & 0.29 & 0.18 \\
\hline $5 p \pi, v=3$ & 840.17 & -0.88 & 5.56 & 0.033 & 0.042 & 2.08 & 1.18 & 99 & 4.43 & 6.23 \\
\hline $31 p 1, v=1$ & 842.81 & -0.39 & 9.06 & 0.22 & 0.14 & 0.18 & 0.41 & 100 & 2.53 & 2.16 \\
\hline $8 p 1, v=2$ & 847.51 & -0.09 & 16.60 & 0.18 & 0.18 & 0.085 & 0.105 & 100 & 0.98 & 0.63 \\
\hline $17 p 3, v=1$ & 852.25 & -0.05 & 24.94 & $\approx 0.03$ & 0.018 & 0.097 & 0.074 & 100 & $\approx 0.19$ & 0.11 \\
\hline $40 p 1, v=1$ & 888.06 & 0.00 & 25.31 & $\approx 0.09$ & 0.11 & 0.041 & 0.059 & 100 & $\approx 0.24$ & 0.094 \\
\hline $18 p 3, v=1$ & 890.70 & -0.07 & 27.22 & $\approx 0.08$ & 0.043 & 0.034 & 0.048 & 100 & $\approx 0.17$ & 0.080 \\
\hline $41 p 1, v=1$ & 893.46 & -0.07 & 31.89 & $\leq 0.02$ & 0.007 & 0.036 & 0.042 & 100 & 0.046 & 0.039 \\
\hline $7 p 3, v=2$ & 983.58 & -0.15 & 6.95 & 0.17 & 0.17 & 0.40 & 0.33 & 100 & 4.34 & 6.02 \\
\hline$Q(3) 14 p 3, v=1$ & 125678.97 & -0.07 & 14.07 & $\leq 0.04^{\mathrm{a}}$ & 0.036 & 0.37 & 0.28 & 100 & 0.68 & 0.65 \\
\hline $15 p 3, v=1$ & 749.82 & -0.07 & 15.07 & $0.06^{\mathrm{a}}$ & 0.030 & 0.28 & 0.27 & 100 & 0.77 & 0.55 \\
\hline $16 p 3, v=1$ & 807.90 & -0.05 & 16.07 & $\leq 0.04^{\mathrm{a}}$ & 0.024 & 0.23 & 0.24 & 100 & 0.42 & 0.47 \\
\hline $17 p 3, v=1$ & 856.05 & -0.06 & 17.06 & $\leq 0.04^{\mathrm{a}}$ & 0.021 & 0.22 & 0.26 & 100 & 0.40 & 0.41 \\
\hline $18 p 3, v=1$ & 896.45 & -0.06 & 18.04 & $\leq 0.04^{\mathrm{a}}$ & 0.018 & 0.16 & 0.23 & 100 & 0.29 & 0.37 \\
\hline
\end{tabular}


TABLE III. (Continued.)

\begin{tabular}{|c|c|c|c|c|c|c|c|c|c|c|}
\hline \multirow[b]{2}{*}{ Assignment } & \multicolumn{2}{|c|}{$\frac{v}{c}\left(\mathrm{~cm}^{-1}\right)$} & \multirow{2}{*}{$\begin{array}{c}n^{*} \\
\text { Calc. }\end{array}$} & \multicolumn{2}{|c|}{$\frac{\Gamma}{h c}\left(\mathrm{~cm}^{-1}\right)$} & \multicolumn{2}{|c|}{$\sigma_{i}^{(\max )}\left(10^{-16} \mathrm{~cm}^{2}\right)$} & \multirow{2}{*}{$\begin{array}{c}\frac{\sigma_{i}}{\sigma_{a}}(\%) \\
\text { Calc. }\end{array}$} & \multicolumn{2}{|c|}{$A\left(10^{6} \mathrm{~s}^{-1}\right)$} \\
\hline & Obs. & Obs.-calc. & & Obs. & Calc. & Obs. & Calc. & & Obs. & Calc. \\
\hline $19 p 3, v=1$ & 930.66 & -0.06 & 18.97 & $\leq 0.03^{\mathrm{a}}$ & 0.015 & 0.22 & 0.22 & 100 & 0.30 & 0.35 \\
\hline $20 p 3, v=1$ & 959.88 & -0.06 & 19.38 & $\leq 0.02^{\mathrm{a}}$ & 0.013 & 0.29 & 0.28 & 100 & 0.27 & 0.43 \\
\hline $5 p \pi, v=3$ & 976.95 & -0.16 & 5.11 & $\leq 0.04^{\mathrm{a}}$ & $0.003^{\mathrm{b}}$ & 3.88 & 10.31 & $87^{\mathrm{b}}$ & $\ldots$ & 13.54 \\
\hline $21 p 3, v=1$ & 985.22 & n.o. & 18.32 & $\ldots$ & 0.011 & $\ldots$ & 0.057 & 100 & $\ldots$ & 0.021 \\
\hline Mean deviation & & -0.13 & & & & & & & & \\
\hline
\end{tabular}

a Evaluated according to $\Gamma=\left[\Gamma_{o b s}^{2}-\Gamma_{r e s}^{2}\right]^{1 / 2}$ where $\Gamma_{\text {res }}=0.04 \mathrm{~cm}^{-1}$ is effective the resolution width.

${ }^{\mathrm{b}}$ From Ref. 11. These levels have been shown to decay primarily by molecular fluorescence.

ionization quantum yield and the fraction $n_{N^{\prime \prime}}$ (from Table I) of molecules present in the sample that have the particular $N^{\prime \prime}$ value. Column 10 of Tables II and III contains the Einstein $A$ coefficients that can be recalculated by use of Eq. (14). These are only rough estimates as already stated, because of the numerous approximations that have been made.

- Column 11 (last column). Finally, the last column of Tables II and III lists the theoretical value of the Einstein $A$ coefficient for each transition, evaluated here by means of discrete-level expression (11), corresponding to omission of all open channels from the linear algebraic system, Eq. (3). This procedure neglects any transfer of intensity from the quasidiscrete level to the surrounding continua or vice versa. The comparison of the $A$ values listed in columns 10 and 11 of Tables II and III provides an a posteriori rough check of the consistency of our analysis of the data at hand. We consider agreement to within, say, a factor of 2 as satisfactory. For many of the resonances listed in the tables, such agreement is indeed obtained. Exceptions are the resonances which are strongly predissociated and are hardly visible in the photoionization spectrum. In these cases, relation equation (14) is obviously inadequate.

The semi-logarithmic plot of Fig. 11 illustrates this aspect. The figure displays the observed (black) and calculated (red) peak-integrated photoionization intensities (Einstein $A$ coefficients) from the two last columns of Table III for the $P(3)$ and $Q(3)$ transitions, corresponding to $N=2^{c}$ and $N=3^{d}$ upper levels, respectively. The figure shows strikingly how the transition intensities to $d$ symmetry levels exhibit fewer perturbations and overall a smoother behavior than the transitions to the heavily perturbed $c$ symmetry levels. The figure demonstrates further that the agreement with theory improves with increasing observed intensities. The peak-integrated intensities of the weak $P(3)$ transitions, which belong to the Hund's case $d$ Rydberg series $n p 1, v^{+}=1$ with $n \approx 20-40$ (cf. Table III), are nearly systematically observed much stronger than calculated, whereas the agreement is much better for the strong $n p 3, v^{+}=$ 1 interlopers with $n \approx 14-18$. By contrast, inspection of Table III indicates that the observed and calculated peak maxima are in much better agreement. The reason for this no doubt stems from the failure of Eqs. (14) and (11) which we have used to estimate the experimental and theoretical integrated peak intensities. Both expressions neglect the transfer of intensity between the continuum and the discrete level, a transfer which in relative terms will affect weak peaks more than strong ones. Despite these shortcomings, Fig. 11 is seen to provide overall a correct account of the observed and calculated peak intensities.

\section{Competition between ionization and dissociation}

The comparison of the intensities of peaks that appear both in the transmission and in the photoionization spectrum, in principle, provides information about the competition of the photodissociation and photoionization processes. However, Fig. 4 (lower panel) shows that only few absorption transitions clearly stand out above the noise level in the present experiment — cf. also the discussion of this point in Sec. IV A. Unlike in the spectra of Refs. 10 and 11 taken in a roomtemperature gas cell where an absolute calibration has been possible, this is not the case here because we have calibrated the spectra via a comparison with the theoretical spectra, and where moreover we have done this separately for absorption and ionization spectra. An advantage of the present experiment however is its higher resolution and reduced Doppler width, which allows us to map out even narrow line profiles in detail,

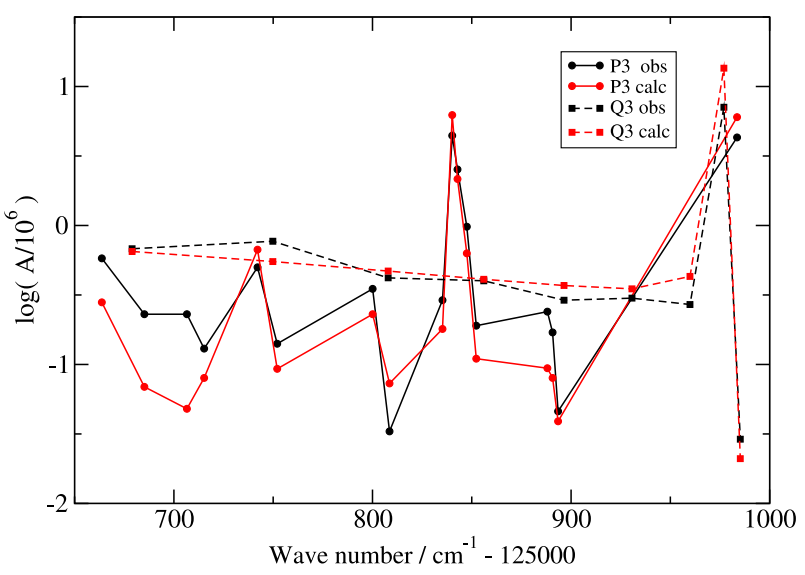

FIG. 11. Integrated peak intensities (Einstein $A$ values) for $P(3)$ (circles connected by full lines) and $Q(3)$ (squares connected by broken lines) transitions. Observed values (black symbols) have been evaluated using Eq. (14), whereas theoretical MQDT values have been evaluated using Eq. (11). The data are presented as a semi-log plot versus the excitation energy. 


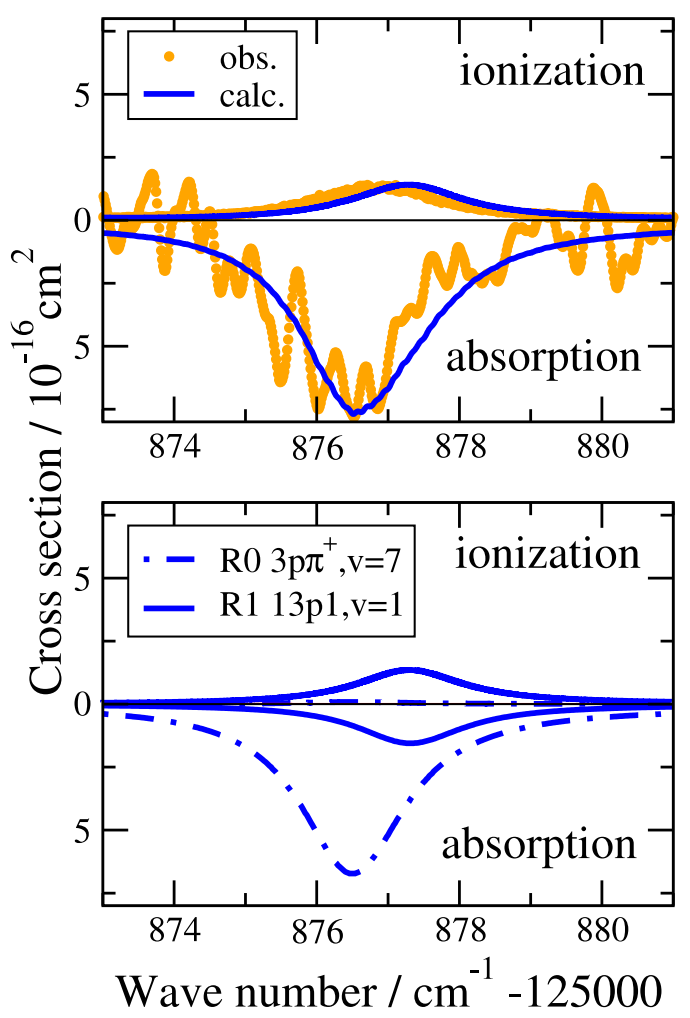

FIG. 12. Photoabsorption and photoionization cross sections of $\mathrm{H}_{2}$ near $125875 \mathrm{~cm}^{-1}$. The absorption is plotted towards negative values on the abscissa scale, whereas ionization is plotted towards positive values. Upper panel—filled dots (orange): experimental values. Full lines (blue): theoretical calculation involving transitions from the $N^{\prime \prime}=0$ and $1 v^{\prime \prime}=0$ ground state levels. Lower panel—dashed-dotted and full blue lines: calculated $R(0)$ and $R(1)$ absorption and ionization profiles shown separately. The upper level of the $R(0) 3 p \pi^{+}, v=7$ is seen to be fully predissociated whereas the $R(1) 13 p 1, v=1$ resonance is fully autoionized (cf. the text for more details).

whereas previously ${ }^{10,11}$ the determination of the competition between the competing decay routes remained limited to peakintegrated information. As an example, we consider here the $R(1)$ transition to the " $6 p \pi, v=2$ " level, cf. Table II, for which we have (in $10^{-16} \mathrm{~cm}^{2}$ )

$$
\begin{aligned}
& \sigma_{a}^{(\max )} \text { (obs.) }=38.76, \quad \sigma_{i}^{(\max )} \text { (obs.) }=0.96, \\
& \sigma_{a}^{(\max )}(\text { calc. })=28.45, \quad \sigma_{i}^{(\max )}(\text { calc. })=0.90 .
\end{aligned}
$$

The agreement is reasonable considering the approximate nature of our intensity calibrations. We stress once again that the novel aspect here is our ability to test the quantum yields directly based on the cross section maxima.

Another example of the competition between ionization and dissociation processes is illustrated by Fig. 12 which displays the absorption and ionization cross sections (plotted toward negative and positive values, respectively) near $125876 \mathrm{~cm}^{-1}$ where the strong $3 p \pi D, v=7 R(0)$ resonance appears. Superposed on this resonance is the $13 p 1, v=1 R(1)$ resonance, the intensity maximum of which differs only by $0.6 \mathrm{~cm}^{-1}$ from that of $3 p \pi D, v=7 R(0)$, that is, by less than the widths of the two resonances, which are about $2 \mathrm{~cm}^{-1}$ in each case. The two resonances can therefore not be distinguished in absorption. However, because the $3 p \pi D, v=7, N=1^{c}$ upper level is fully predissociated, it does not appear in the photoionization spectrum, so that the
TABLE IV. Unidentified lines in the photoionization spectrum of $\mathrm{H}_{2} . v / c$, transition wave number. $\sigma_{i}^{(\max )}$, ionization cross section at peak maximum. $\Gamma / h c$, resonance width (FWHM). Peaks for which no width is given have a width $\leq 0.04 \mathrm{~cm}^{-1}$.

\begin{tabular}{rcc}
\hline \hline$\frac{v}{c}\left(\mathrm{~cm}^{-1}\right)$ & $\sigma_{i}^{(\max )}\left(10^{-16} \mathrm{~cm}^{2}\right)$ & $\frac{\Gamma}{h c}\left(\mathrm{~cm}^{-1}\right)$ \\
\hline 125607.36 & 0.14 & 0.25 \\
629.83 & 0.35 & $\ldots$ \\
650.27 & 0.73 & $\ldots$ \\
672.21 & 0.09 & $\approx 0.1$ \\
672.87 & 0.13 & $\approx 0.5$ \\
686.43 & 0.25 & $\ldots$ \\
707.63 & 0.26 & $\ldots$ \\
739.01 & 0.04 & $\approx 0.8$ \\
798.72 & 0.10 & $\ldots$ \\
811.53 & 0.12 & $\ldots$ \\
839.41 & 0.06 & $\ldots$ \\
864.06 & 0.23 & $\ldots$ \\
937.82 & 0.08 & $\ldots$ \\
939.93 & 0.04 & $\ldots$ \\
960.42 & 0.24 & $\ldots$ \\
964.00 & 0.14 & \\
\hline \hline
\end{tabular}

$13 p 1, v=1, N=2^{c}$ resonance appears in this spectrum at a slightly shifted cross section maximum. The observed and calculated absorption and ionization cross section profiles agree well on the absolute scales given in Fig. 12, thus confirming the correctness of the decay dynamics predicted by MQDT.

\section{Unidentified lines}

Table IV lists a number of lines that appear in the photoionization spectrum which remain unidentified at this point. They could correspond to $R(3)$ transitions or transitions with $N^{\prime \prime}>3$ leading to the $n p$ manifold of excited states which have not been included in the present calculations. Alternatively they could also arise from the $n f$ manifold of states, although these are expected to be very weak in ground state absorption as they would correspond to a nominally (but not strictly) forbidden $\Delta \ell=3$ transition. We leave their identification to future work.

\section{CONCLUSIONS}

The widths of known Feshbach autoionization/predissociation resonances of $\mathrm{H}_{2}$ in the vicinity of the ionization threshold span the range from $\approx 0.0005$ to $\approx 50 \mathrm{~cm}^{-1}$, that is, no less than five orders of magnitude. Among these, the natural profiles of the longest-lived resonances may be inferred only indirectly from their absorption intensities ${ }^{10}$ [cf. Eq. (12)] or directly by lifetime measurements. ${ }^{43}$ On the other hand, the profiles of the resonances with the shortest lifetimes are so broad that they hardly emerge from the continuum background and are also difficult to observe. An example for this latter situation is offered by the $3 p \pi D, v=7 R(2)$ transition near $125720.6 \mathrm{~cm}^{-1}$ (see Table III), which is just barely visible in the theoretical dissociation cross section shown in Fig. 5. 
In the present work, the effective spectral resolution has been improved by more than an order of magnitude as compared to all previous $\mathrm{H}_{2}$ ground state absorption and photoionization studies, by reducing the Doppler width and using a high-resolution VUV laser system. As a result, for instance, the natural line profiles of the $v=1 Q\left(N^{\prime \prime}\right)$ transitions that autoionize via a $\Delta v=1$ process have become accessible to direct observation for the first time.

The experimental and theoretical photoionization spectra displayed in Figs. 7-10 provide the principal ground for the comparison of the present experiments and our first-principles MQDT calculations. It is the direct visual comparison of the experimental and theoretical cross section profiles which allows us to assess best to what extent the rich and irregular photoionization structures are reproduced by theory. For this reason also it has not been necessary to invoke the asymmetric Beutler-Fano resonance profile, ${ }^{21}$ although many of the resonances observed in this work may be represented approximately by the corresponding formula. ${ }^{44}$ Complementary information, in particular, concerning the peak heights, which are not always visible in the figures, is contained in numerical form in Tables II and III. Integrated peak intensities given in the form of Einstein $A$ coefficientsas opposed to peak maxima-are also of interest and are listed in the tables along with the resonance widths. However, these quantities have no strict conceptual meaning, and their analysis has deliberately been left here at a relatively rough stage.

\section{ACKNOWLEDGMENTS}

We thank Professor H.-J. Wörner (Zürich) for help with the MQDT calculations during an early stage of this work. This work is supported financially by the Swiss National Science Foundation and the ETHZürich. J.Zs.M. is grateful for financial support from the Triangle de la Physique (SpeCoRyd contract) and the LabeX EMC3 (PicoLIBS project). Ch.J. is grateful to the Ernst Miescher Foundation (Basel, Switzerland) for partial support.

${ }^{1}$ G. Herzberg, J. Mol. Spectrosc. 33, 147 (1970).

${ }^{2}$ G. Herzberg and Ch. Jungen, J. Mol. Spectrosc. 41, 425 (1972).

${ }^{3}$ W. Kołos and L. Wolniewicz, J. Chem. Phys. 49, 404 (1968).

${ }^{4}$ G. Hunter and H. O. Pritchard, J. Chem. Phys. 46, 2153 (1967).

${ }^{5}$ S. Takezawa, J. Chem. Phys. 52, 2575 (1970).

${ }^{6}$ U. Fano, Phys. Rev. A 2, 353 (1970).

${ }^{7}$ P. M. Dehmer and W. A. Chupka, J. Chem. Phys. 65, 2243 (1976).
${ }^{8}$ M. Rothschild, H. Egger, R. T. Hawkins, J. Bokor, H. Pummer, and C. K. Rhodes, Phys. Rev. A 23, 206 (1981).

${ }^{9}$ G. D. Dickenson, T. I. Ivanov, M. Roudjane, N. de Oliveira, D. Joyeux, L. Nahon, W.-Ü. L. Tchang-Brillet, M. Glass-Maujean, I. Haar, A. Ehresmann, and W. Ubachs, J. Chem. Phys. 133, 144317 (2010).

${ }^{10}$ M. Glass-Maujean, Ch. Jungen, H. Schmoranzer, A. Knie, I. Haar, R. Hentges, W. Kielich, K. Jänkälä, and A. Ehresmann, Phys. Rev. Lett. 104, 183002 (2010).

${ }^{11}$ M. Glass-Maujean, Ch. Jungen, G. Reichardt, A. Balzer, H. Schmoranzer, A. Ehresmann, I. Haar, and P. Reiss, Phys. Rev. A 82, 062511 (2010).

${ }^{12}$ M. Glass-Maujean, Ch. Jungen, H. Schmoranzer, I. Tulin, A. Knie, P. Reiss, and A. Ehresmann, J. Mol. Spectrosc. 293-294, 19 (2013).

${ }^{13}$ Ch. Jungen, S. T. Pratt, and S. C. Ross, J. Phys. Chem. 99, 1700 (1995).

${ }^{14}$ J. K. G. Watson, J. Mol. Spectrosc. 145, 130 (1991).

${ }^{15}$ A. de Shalit and H. Feshbach, Nuclear Physics: Nuclear Structure (Wiley, New York, 1974).

${ }^{16}$ E. R. Davidson, J. Chem. Phys. 35, 1189 (1961).

${ }^{17}$ M. Sommavilla, U. Hollenstein, G. M. Greetham, and F. Merkt, J. Phys. B: At. Mol. Opt. Phys. 35, 3901 (2002).

${ }^{18}$ U. Hollenstein, H. Palm, and F. Merkt, Rev. Sci. Instrum. 71, 4023 (2000).

${ }^{19}$ U. Hollenstein, R. Seiler, H. Schmutz, M. Andrist, and F. Merkt, J. Chem. Phys. 115, 5461 (2001).

${ }^{20}$ M. J. Seaton, Rep. Prog. Phys. 46, 167 (1983).

${ }^{21} \mathrm{U}$. Fano and A. R. P. Rau, Atomic Collisions and Spectra (Academic, Orlando, 1986).

${ }^{22}$ Ch. Jungen, "Elements of quantum defect theory," in Handbook of High Resolution Spectroscopy, edited by M. Quack and F. Merkt (Wiley, Chichester, New York, 2011).

${ }^{23}$ C. H. Greene and Ch. Jungen, Adv. At. Mol. Phys. 21, 51 (1985).

${ }^{24}$ R. Guérout, Ch. Jungen, H. Oueslati, S. C. Ross, and M. Telmini, Phys. Rev. A 79, 042717 (2009).

${ }^{25}$ F. S. Ham, Solid State Phys. 1, 127 (1955).

${ }^{26}$ Ch. Jungen and G. Raseev, Phys. Rev. A 57, 2407 (1998).

${ }^{27}$ J. Zs. Mezei, I. F. Schneider, M. Glass-Maujean, and Ch. Jungen, J. Chem. Phys. 141, 064305 (2014).

${ }^{28}$ L. Wolniewicz and G. Staszewska, J. Mol. Spectrosc. 212, 208 (2002).

${ }^{29}$ L. Wolniewicz and G. Staszewska, J. Mol. Spectrosc. 220, 45 (2003).

${ }^{30} \mathrm{~A}$ compilation of the numerical data published in Refs. 28 and 29 can be found in http://www.fizyka.umk.pl/publications/ifiz/luwo/.

${ }^{31} \mathrm{Ch}$. Jungen and O. Atabek, J. Chem. Phys. 66, 5584 (1977).

${ }^{32}$ M. Glass-Maujean and Ch. Jungen, J. Phys. Chem. A 113, 13124 (2009).

${ }^{33}$ Ch. Jungen, Phys. Rev. Lett. 53, 2394 (1984).

${ }^{34}$ Ch. Jungen and S. C. Ross, Phys. Rev. A 55, R2503 (1997).

${ }^{35}$ A. Matzkin, Ch. Jungen, and S. C. Ross, Phys. Rev. A 62, 062511 (2000).

${ }^{36}$ H. Wind, J. Chem. Phys. 42, 2371 (1965).

${ }^{37}$ D. M. Bishop and R. W. Wetmore, Mol. Phys. 26, 145 (1973); 27, 279 (1974).

${ }^{38}$ L. Wolniewicz and T. Orlikowski, Mol. Phys. 74, 103 (1991).

${ }^{39}$ L. Wolniewicz, J. Chem. Phys. 99, 1851 (1993).

${ }^{40}$ L. Wolniewicz, J. Chem. Phys. 103, 1792 (1995).

${ }^{41}$ K. Pachucki and J. Komasa, J. Chem. Phys. 143, 034111 (2015).

${ }^{42}$ M. Glass-Maujean, A.-M. Vasserot, Ch. Jungen, H. Schmoranzer, A. Knie, S. Kübler, and A. Ehresmann, J. Mol. Spectrosc. 315, 155 (2015).

${ }^{43}$ M. Glass-Maujean, J. Breton, B. Thièblemont, and K. Ito, J. Phys. 45, 1107 (1984).

${ }^{44}$ U. Fano, Phys. Rev. 124, 1866 (1961). 